\title{
Die „Riesenhirschfundstelle“ von Endingen: geowissenschaftliche und archäologische Untersuchungen an einem spätglazialen Fundplatz in Vorpommern')
}

\author{
Knut Kaiser, Pim de Klerk \& Thomas Terberger*) \\ - elk, giant deer, Bölling, Alleröd, palynostratigraphy, sedimentation, Vorpommern, \\ Northeastern Germany -
}
Inhalt
1 Einführung
2 Ältere stratigraphische Beobachtungen
3 Geologisch-bodenkundliche Verhältnisse
4 Vegetationsrekonstruktion
5 Radiokarbondaten
6 Archäologische Ergebnisse
7 Megaloceros giganteus im Spätglazial des nördlichen Mitteleuropa
8 Zusammenfassende Diskussion
9 Schriftenverzeichnis

Kurzfassung: Vorgestellt werden Neuuntersuchungen an einem 1899 beim Sandabbau entdeckten spätglazialen Fundplatz im Kr. Nordvorpommern. Die z. T. Bearbeitungsspuren aufweisenden Knochenartefakte stammen zum größten Teil vom Elch, je einmal sind Riesenhirsch und Pferd vertreten. Lithische Artefakte sind nicht überliefert. Der in das frühe Alleröd datierende Elchjägerplatz Endingen VI repräsentiert den ältesten absolut datierten Nachweis einer menschlichen Besiedlung in Nordostdeutschland. Die Fundschicht selbst ist offenbar vollständig zerstört worden. Unmittelbar benachbarte Profile weisen eine Abfolge basaler Geschiebemergel, fluvialer Sand des Pleniglazials, spätglaziale Silikatmudde und fluvialer Sand der Jüngeren Dryas auf. Ein Pollendiagramm aus der Silikatmudde zeigt eine Palynostratigraphie vom „Bölling“ (neu: „Hippophaë-Phase“) bis zum mittleren Alleröd. Die Sedimentbildung und die Vegetationsentwicklung am Fundplatz werden erläutert sowie spätglaziale Nachweise des Riesenhirsches im nördlichen Mitteleuropa diskutiert.

\section{[The "giant deer-site" of Endingen: geoarchaeolo- gical investigations at a Lateglacial site in Vor- pommern (Northeastern Germany)]}

Abstract: This text introduces recent investigations of a Lateglacial archaeological site in the district Nordvor-

1) Herrn Prof. Dr. Wolfgang Janke/Greifswald zum 65. Geburtstag gewidmet.

*) Anschrift der Verfasser: Dipl.-Geogr. K. KaISER, Dipl.Geogr. P. DE KLERK, Universität Griefswald, Geographisches Institut, Friedrich-Ludwig-Jahn-Straße 16, D-17487 Greifswald, Dr. Th. Terberger, Universität Greifswald, Historisches Institut, Lehrstuhl für Ur- und Frühgeschichte, Hans-Fallada-Straße 1, D-17489 Greifswald pommern (NE Germany), which was discovered in 1899 in a sandpit. The bones, which partly show traces of human use, originate mainly from elk; single finds belong to giant deer and horse. Stone artefacts have not been found. This elk-hunter-camp Endingen VI, which is dated in the early Alleröd, represents the oldest known absolutely dated human settlement in NE Germany. The findlayer itself probably has been completely destroyed. Neighbouring profiles show a succession of basal till, Pleniglacial fluvial sands, Lateglacial muds and fluvial sands of the Younger Dryas. A Pollendiagram from the muds shows a palynostratigraphy ranging from the "Bölling" (new: "Hippophaë-phase") up to the middle Alleröd. Sedimentation and vegetational development of the site are discussed, as well as Lateglacial evidence of giant deer in northern CentralEurope.

\section{Einführung}

In einem Grundmoränenbecken westlich von Stralsund unweit des Gutes Endingen, Kr. Nordvorpommern, kamen 1899 beim Sandabbau teilweise bearbeitete Faunenreste zum Vorschein (Abb. 1, 2). Nach einer knappen Vorlage des Materials durch DeEcke (1900a, b) und ersten geowissenschaftlichen Arbeiten durch Gross (1938, 1954) mit dem Ergebnis einer Datierung des Endinger Materials vor die spätweichselzeitliche „Vegalster Staffel“ wurde durch WoldstedT (1955) ein „Interglazial von Endingen“ postuliert. Das Material und die Fundstelle gerieten danach aber weitgehend in Vergessenheit und so fehlte bislang eine verläßliche Bearbeitung des geowissenschaftlich interessanten Fundplatzes. Aus archäologischer Perspektive erhält dieser seine überregionale Bedeutung durch die Seltenheit absolut datierbarer archäologischer Inventare aus dem Spätglazial. Im Rahmen eines Projektes innerhalb des DFG-Schwerpunktprogrammes „Wandel der Geobiosphäre während der letzten 15.000 Jahre“ (vgl. Billwitz et al. 1998) konnte der Fundplatz in den vergangenen Jahren umfassend neu untersucht werden. Die Arbeiten galten einer archäologischen und archäozoologischen Neubearbei- 

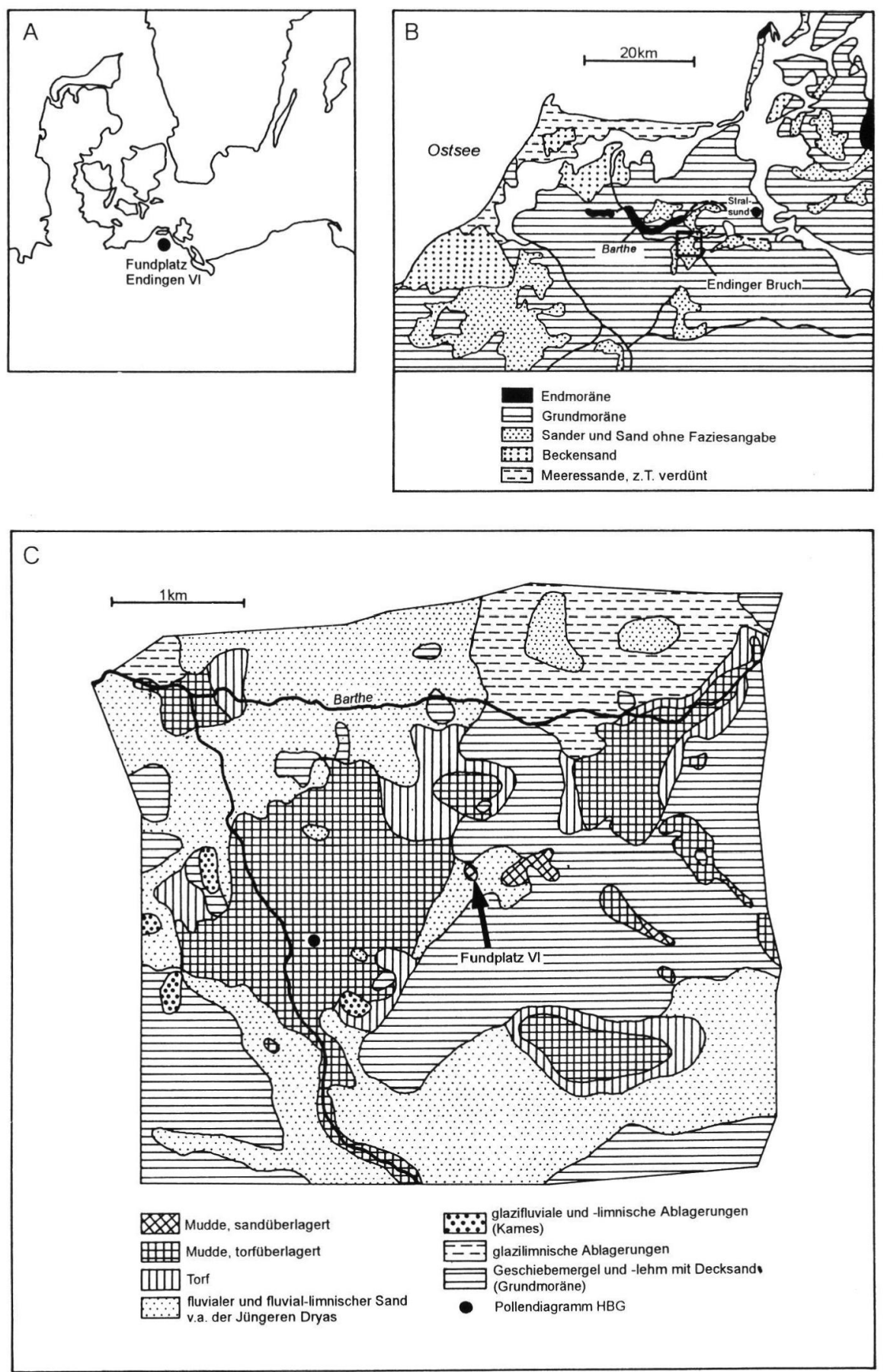

Abb. 1: A = Lage des Fundplatzes Endingen VI, B = Quartärgeologie im nördlichen Vorpommern (Quelle: Geologische Karte von Mecklenburg-Vorpommern 1:500 000, Schwerin 1994, verändert), C = vereinfachte geologische Kartierung des Endinger Bruchs und seiner Umgebung.

Fig. 1: $\mathrm{A}=$ location of site Endingen VI, $\mathrm{B}=$ Quaternary geology in northern Vorpommern, $\mathrm{C}=$ simplified geological map of the area around the site. 


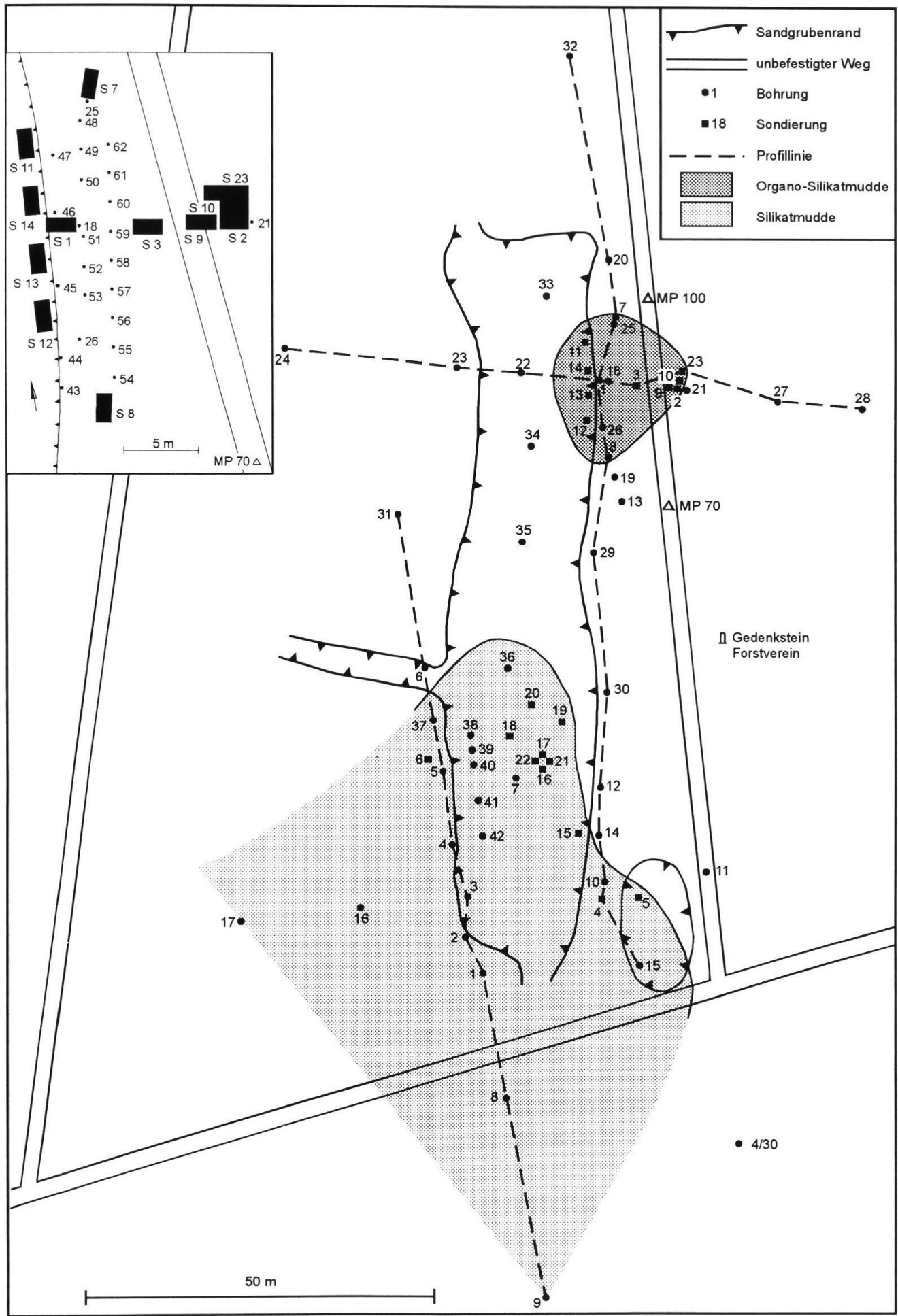

Abb. 2: Fundplatz Endingen VI mit Bohrungen,. Sondagen, Schnittspuren und spätglazialen Muddearealen.

Fig. 2: Fundplatz Endingen VI with corings, exploration pits, sections and Lateglacial mud-areas. 
tung sowie radiometrischen Datierungen der Altfunde, der Lokalisierung der Fundschicht und Untersuchungen zur Stratigraphie der Fundstelle. Nach Vorlage der archäologischen Ergebnisse durch Terberger (1996a) und STREeT (1996) stehen im folgenden die geowissenschaftlichen Aspekte im Vordergrund.

\section{2 Ältere stratigraphische Beobachtungen}

Ein durch DEECKE (1900a: 2f) aufgenommenes Profil bestand aus einem hangenden, z. T. kiesigen Sand mit Geschiebeblöcken und einem liegenden „nicht durchsunkenen, aber ziemlich mächtigen... Torfschlick und schwärzlich blauen Ton“ (Abb. 3). Die Riesenhirschreste, die Knochen von Hecht und Ente sowie die botanischen Reste stammen aus dem „Torfschlick“, die Elchreste sollen aus den hangenden Sanden stammen. Nach einem modernen Verständnis des Wortes „Torfschlick“ könnte es sich bei diesem Sediment um eine Gyttja bzw. eine Mudde im Sinne eines Flachwasserabsatzes handeln.

Für die Datierung des Profils und der Funde nutzte DeEcke (1900a: 3) geologische Argumente und deutete den hangenden Kiessand als Produkt „der Thätigkeit von Gletscherbächen": Die Bildungen müssten demnach an der Grenze von Diluvium und Alluvium stehen, also altalluvial sein." Unter Berücksichtigung der damaligen Nachweise des Riesenhirsches wird auch ein, wenngleich nach DeEckes eigenen Worten kaum glaubhaftes ,interglaciales" Alter diskutiert (DeEcke $1900 \mathrm{a}$ : 9). Durch Gross (1938) wurde ein Profil mit der Abfolge liegender toniger Sand, Dy-Tongyttja und hangender blockführender Kies auf der Ostseite im nördlichen Teil der Sandgrube untersucht. Nach Trautzsch (1958) ging ein offenbar vorhandener umfangreicher Untersuchungsbericht von Gross inklusive Pollenprofil durch Kriegseinwirkungen verloren. Faßt man die Angaben von Gross (1938, 1954, 1958) zusammen, werden für die Endinger Riesenhirschfundstelle folgende Aussagen getroffen: Das untersuchte Dy-Tongyttjavorkommen im Nordosten der Sandgrube ist Bestandteil eines größeren spätglazialen Gewässers, wobei die eigentliche Faunen-Fundstelle von 1899 jedoch im Süden der Grube vermutet wird. Die Mudde der Fundschicht wird zunächst in die Ältere Dryas/den Übergang zum Alleröd, später hingegen in die Älteste Dryas datiert. Das Hangende wird, der späteren Datierung folgend, als grober Vorschüttsand der Velgaster Staffel angesprochen. Dieser Interpretation der Endinger Stratigraphie folgt auch WoLDSTEDT (1955: 223).2)
Durch Trautzsch (1958: 34, Abb. 12) wurde im Südbereich der Sandgrube ein etwa 40 x $30 \mathrm{~cm}$ großes, von kiesigen Sanden überlagertes und von Geschiebemergel unterlagertes Vorkommen einer wenige Dezimeter mächtigen ,graugrünen stark sandigen und kalkhaltigen Gyttja" erbohrt. Das Gyttja-Vorkommen wurde chronologisch ,an den Beginn des Gotiglazials in die waldlose Periode des Spätglazials" gestellt und die hangenden Kiessande allgemein der „subarktischen Periode“ des Spätglazials zugewiesen.

\section{Geologisch-bodenkundliche Verhältnisse}

Im Bereich der Fundstelle wurden 62 Peilstangenund Rammkernbohrungen sowie 23 Sondagen niedergebracht (Abb. 4). Die Aufnahme der Profile, die bodenkundliche Terminologie und die Vorschriften für Laboranalysen folgen der AG BODEN (1994) und SCHLichting et al. (1995). Alle \%-Angaben der Bodenanalysen beziehen sich auf Gewichtsprozent (Abb. 5).

Unter einer z. T. mehrere Meter mächtigen, zumeist kiesig-sandigen Deckschicht zeichnen sich, im Gegensatz zu Gross (1938) und Trautsch (1958), zwei getrennte Areale spätglazialer Silikatmudden ab. Teilweise formt die Deckschicht einen stark vom Sandabbau veränderten Flachhügel: eine jüngere, schwach ausgeprägte Vollform folgt einer älteren Hohlform (Abb. 4, mittlerer Schnitt). Während das nördliche Muddevorkommen von ca. 22 x $19 \mathrm{~m}$ durch die Vielzahl von Bohrungen und Sondagen exakt abgrenzbar ist, muß die südliche und westliche Ausdehnung des südlichen Vorkommens offen bleiben. Hier kann aber im Bereich der Sandgrube von einer ehemaligen Ufersituation ausgegangen werden. Für den Fundplatz VI ergibt sich nach den neuen Untersuchungen folgende Lithostratigraphie: Über Geschiebemergel (Einheit 1) folgen pleniglaziale fluviale Sande (Einheit 2), daran schließen sich spätglaziale Stillgewässerablagerungen an (Einheit 3), den Abschluß bilden spätglaziale, sandig-kiesige bzw. sandige Deckschichten fluvialer Genese (Einheit 4).

2) „Interglazial von Endingen. Im Wald von Endingen (Kreis Franzburg-Barth) liegt eine Dytongyttja unter geschichteten groben Sanden und Kiesen mit kopfgroßen Geschieben. Diese Gyttja ist nach der pollenanalytischen Untersuchung von Gross (unveröffentlicht) in einer baumlosen Tundrenzeit abgelagert worden. In ihr wurden Reste von Riesenhirsch (angeblich bearbeitet) und Elch gefunden. Das Hangende dieser Gyttja wird wahrscheinlich von Vorschüttsanden der Velgaster Staffel gebildet." (WOLDSTEDT 1955: 223) 


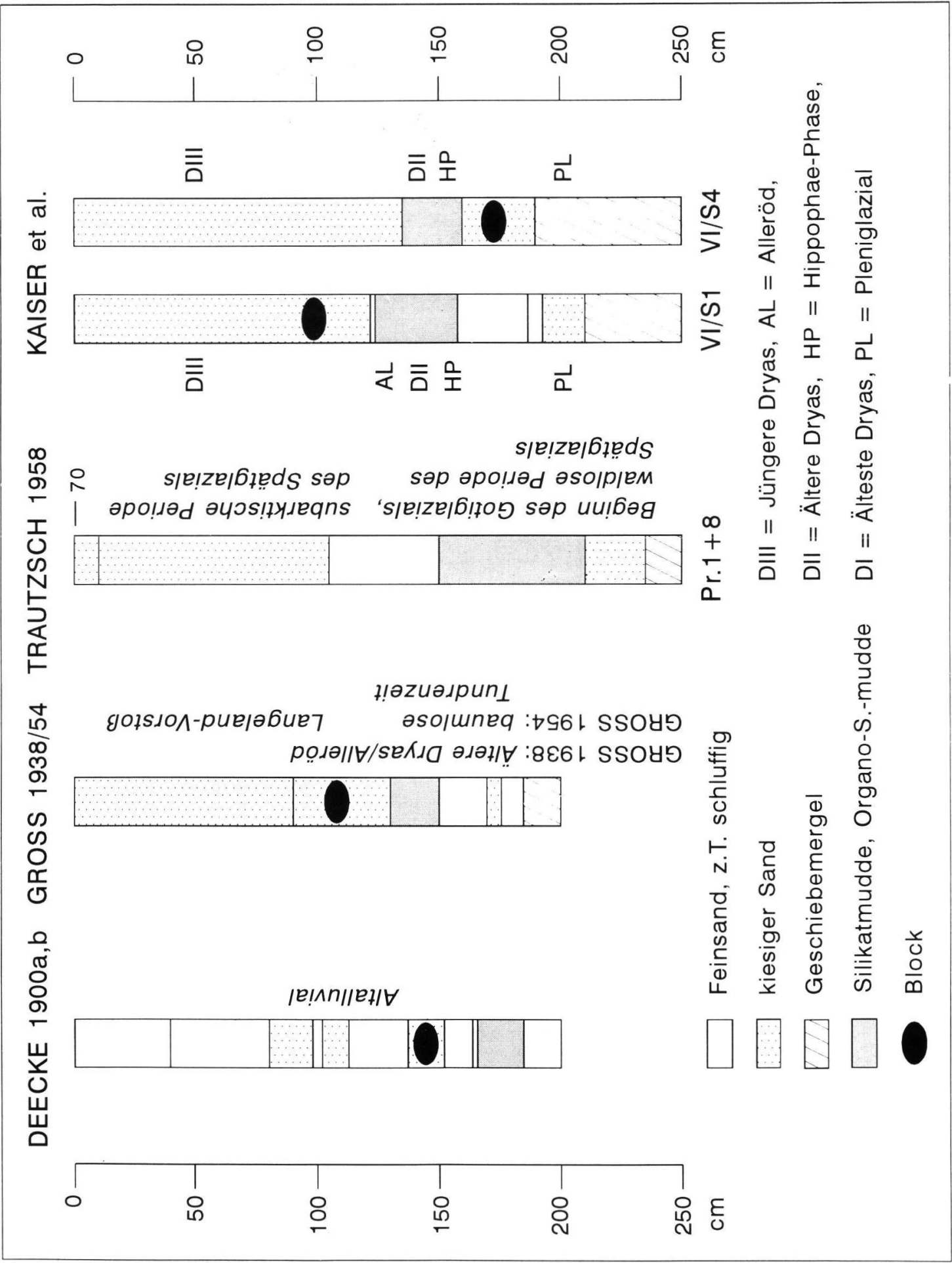

Abb. 3: Stratigraphie des Fundplatzes Endingen VI nach verschiedenen Autoren.

Fig. 3: Stratigraphy of site Endingen VI after various authors. 

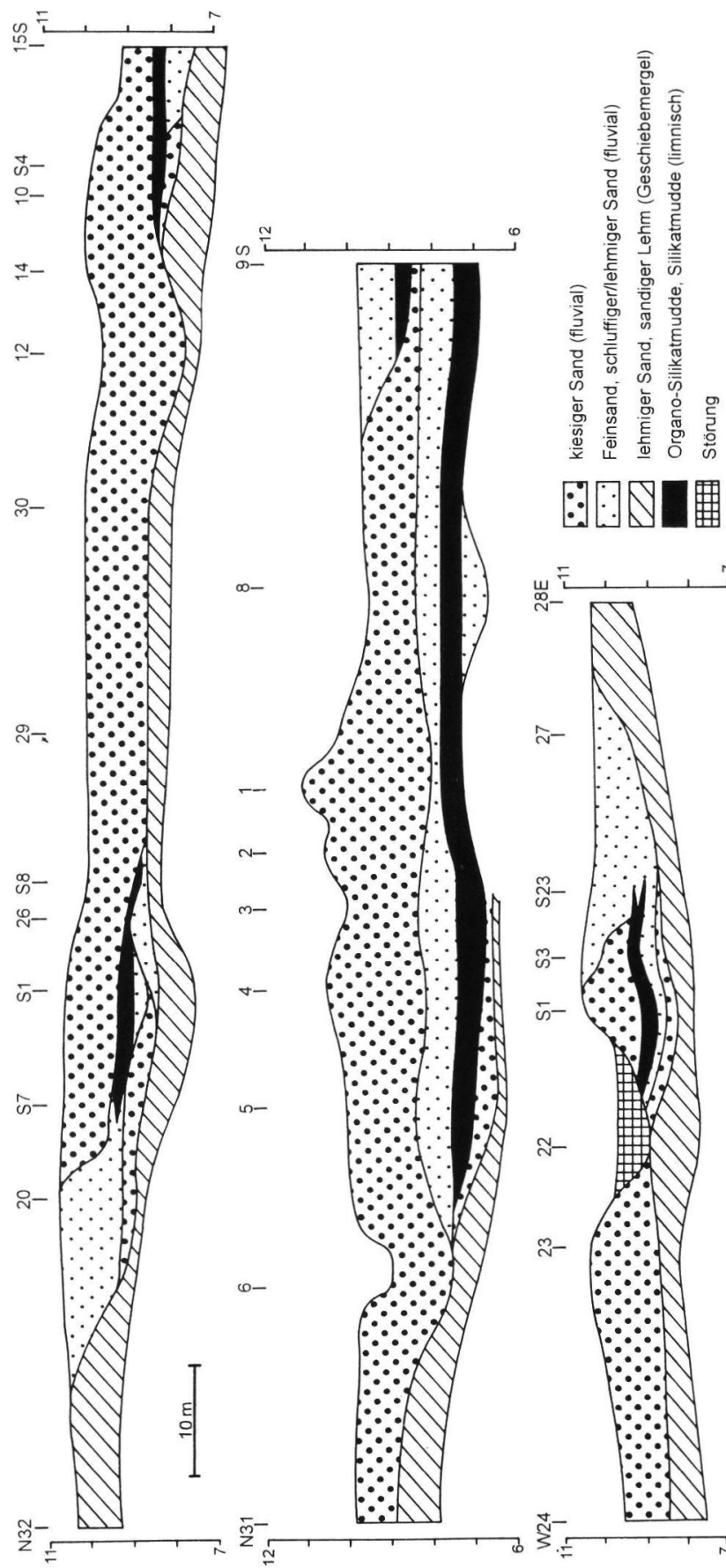

$\underset{\sim}{\stackrel{w}{\sim}-}$

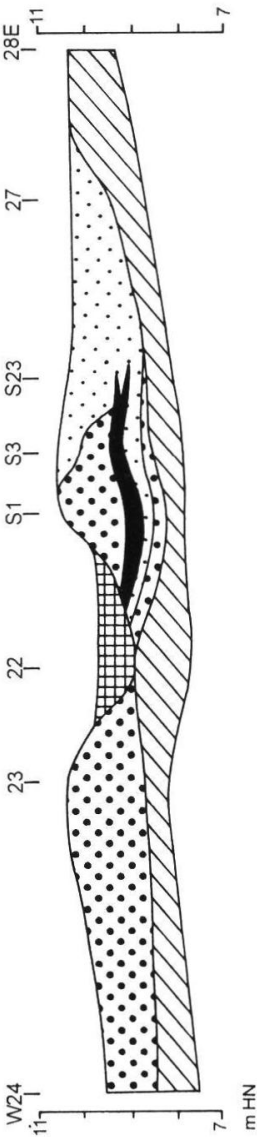

Einheit 1

Geschiebemergel

Der sehr schlecht sortierte Geschiebemergel besteht aus stark lehmigem Sand bis mittelsandigem Lehm und weist einen Carbonatgehalt von $7-13 \%$ auf. Räumlich könnte der sehr sandige Geschiebemergel der Grundmoräne des jüngsten Weichselvorstoßes in Nordostdeutschland, dem „Mecklenburger Vorstoß“ (= ,W3“, vgl. RÜHBERG et al. 1995), entsprechen. Auch in aktuellen Kartenveröffentlichungen (GEOLOGISCHES LANDESAMT M-V 1995) wird das oberste Geschiebemergelstockwerk in der Umgebung des Endinger Bruchs als „W3“ ausgewiesen. Zur Überprüfung dieser stratigraphischen Einstufung wurden an den Profilen $\mathrm{VI} / \mathrm{S} 4\left(\mathrm{CaCO}_{3}=13 \%\right.$, unverwittert) und $\mathrm{VI} / \mathrm{S} 23$ $\left(\mathrm{CaCO}_{3}=7 \%\right.$, evtl. angewittert) Kleingeschiebezählungen nach TGL 25232 (1971) durchgeführt. Mit Geschiebesummen von 836 bzw. 551 Stück pro $5 \mathrm{~kg}$ (entspricht $127 \mathrm{bzw} .110 \mathrm{G} / \mathrm{kg}$ ) ist der deutlich zu hohe Geschiebebestand nicht oder nur mit Vorbehalt interpretierbar. Im Auswertedreieck ergibt sich eine Zuordnung als saalezeitlicher Geschiebemergel. Absolut uncharakteristisch verhält sich bei den Endinger Proben der Quotient aus Nordischem Kristallin und Paläozoischen Kalksteinen mit Werten von 4,4 bzw. 11,2 gebenüber $\leq 1,0$ in $75 \%$

aller untersuchten „W3“-

Abb. 4: Geologische Schnitte am Fundplatz Endingen VI.

Fig. 4: Geological sections at site Endingen VI. 


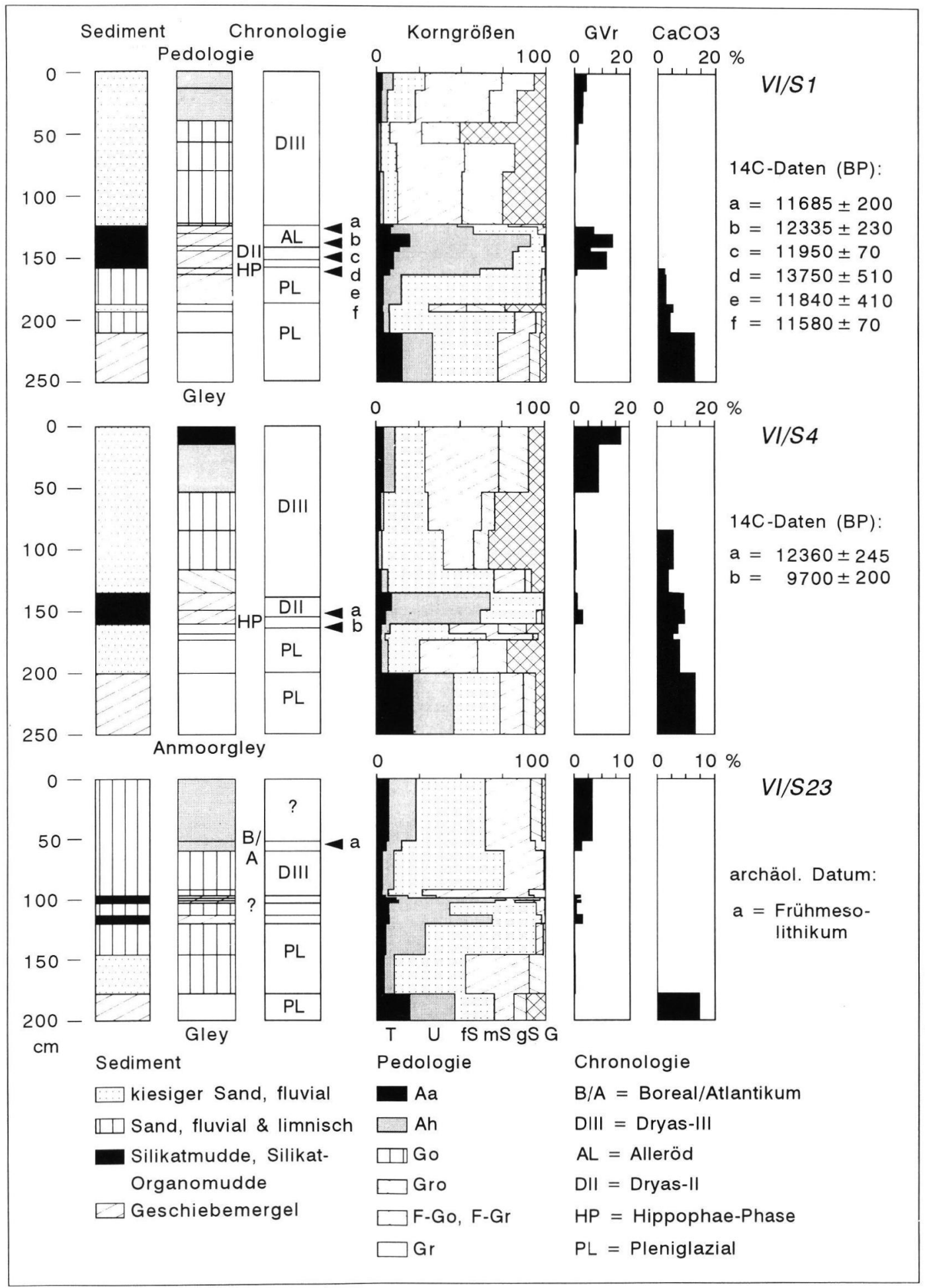

Abb. 5: Sedimentologie, Pedologie und Chronologie der Profile VI/S1, VI/S4 und VI/S23 (GVr = reduzierter Glühverlust $=$ Abzug von 0,1\% des GV je 1\% Ton).

Fig. 5: Sedimentology, pedology and chronology of profiles VI/S1, VI/DS4 and VI/S23 (GVr = reduced loss on ignition). 
an einem spätglazialen Fundplatz in Vorpommern

Proben (vgl. RüHBERG 1987). Auch weitere Quotienten weisen deutliche Abweichungen auf. Die Proben lassen sich daher keinem definierten Geschiebemergel zuordnen; wahrscheinlich liegt ein Problem der Till-Ausprägung vor (lokale Aufnahme fluvialen Materials?).

\section{Einheit 2 - glazifluviale Sande}

In 10 - $70 \mathrm{~cm}$ Mächtigkeit werden die spätglazialen Stillwasserablagerungen von geschichteten, mittel bis sehr schlecht sortierten, z. T. kies- und kalkhaltigen Sanden verschiedener Korngröße (lehmiger Sand bis Grobsand) unterlagert. Teilweise kommen, wie z. B. in VI/S4, Steine und Blöcke vor. Fehlende organische Substanz, fehlende Fossilien und die stratigraphische Position verweisen auf ein pleniglaziales Alter der Sande.

\section{Einheit 3 - Stillgewässerablagerungen}

Es konnten zwei Typen spätglazialer Mudden festgestellt werden. Im nördlichen Teil der Fundstelle (vgl. Abb. 2) eine ca. $35 \mathrm{~cm}$ mächtige feingeschichtete dunkelbraune Organo-Silikatmudde (Profil VI/S1: Schluff $=71-72 \%, \mathrm{GV}=7-25 \%$, $\mathrm{CaCO}_{3}=0$ ), im südlichen Teil eine ca. $25 \mathrm{~cm}$ mächtige, dunkelgraue Silikatmudde (Profil $\mathrm{VI} / \mathrm{S} 4:$ Schluff $=57-59 \%, \mathrm{GV}=2-4 \%, \mathrm{CaCO}_{3}=$ $9-10 \%$ ). Ein weiteres sandig-schluffiges limnisches Sediment unter der Organo-Silikatmudde in Profil VI/S1 ist pollenfossilfrei und datiert in den Zeitraum vor die Älteste Dryas. Diatomeenpräparate aus den Mudden zeigen in VI/S1 Kleinstschill und Nadelreste sowie in VI/23 stark korrodierte und zumeist unbestimmbare Diatomeenreste. Die wenigen bestimmbaren Diatomeen (Analyse: W. JANKE/Greifswald) lassen auf ein flaches und pflanzenreiches Kleingewässer schließen in das silikatische Komponenten schwacher Ufer- und Oberflächenerosion eingetragen wurden. In beiden Muddearealen setzt die spätglaziale limnische Sedimentation in der Hippophaë-Phase ein (vgl. Abschnitt 4). Die Mudden enden im Profil VI/S4 in der Älteren Dryas, im Profil VI/S1 dagegen erst im Alleröd. Das frühere Ende in VI/S4 dürfte auf eine fluviale Erosion der ehemals vorhandenen Alleröd-Sedimente während der Jüngeren Dryas zurückgehen: In VI/S1 schiebt sich zwischen die parallelgeschichtete Mudde des mittleren Alleröds und den hangenen Kiessand eine $6 \mathrm{~cm}$ schmale Lage aus verlagerter Mudde und belegt den fluvialen Abtrag des jüngeren Sedimentes. Die unterschiedliche lithologische Ausprägung der vertikal ca. $1 \mathrm{~m}$ voneinander entfern- ten Mudden in VI/S1 und VI/S4 ist auf unterschiedliche Sedimentationsbedingungen zurückzuführen (Wassertiefe und -bewegung, Makrophytenbesatz, Umgebungseinfluß).

Eine topographisch denkbare ehemalige Verbindung beider Muddeareale ist möglicherweise der fluvialen Erosion in der Jüngeren Dryas zum Opfer gefallen (vgl. Abb. 4, oberer Schnitt). Als 1899 fundlieferndes Sediment kommt nach lithologischen und chronologischen Gesichtspunkten das nördliche Muddevorkommen (Organo-Silikatmudde in VI/S1) in Betracht. Die Schichtung, der torfige Habitus sowie die Datierung der jüngsten Teile der Mudde und eines Riesenhirschrestes in das Alleröd (s. u.) ergeben eine offensichtliche Übereinstimmung mit dem „bänderthonartigen Torfschlick“ DeEckes (1900a: 2f).

\section{Einheit 4 - fluviale Deckschichten}

Die Deckschichten können nur indirekt, d. h. durch liegende allerödzeitliche Sedimente, in die Jüngere Dryas datiert werden. Flächig dominieren schlecht bis sehr schlecht sortierte, geschichtete, kiesige Mittelsande (Abb. 5, Profile VI/S1 und VI/S4). Der Kiesanteil beträgt 7 - $51 \%$. Im Bereich von Profil VI/S23 sind auch schwach lehmige und tonige Sande an der Überdeckung liegender Mudden beteiligt. Hier erbringt eine mesolithische Fundschicht (jüngeres Boreal/älteres Atlantikum) einen alt- bis mittelholozänen terminus ante quem der Überdeckung (Abb. 5). In den mehr oder weniger homogenen kiesigen Sanden finden sich regellos gestreut Steine und z. T. auch Blöcke (max. 40 x 25 cm); Gross (1938) beobachtete Blöcke von $70 \times 35 \mathrm{~cm}$. Für den Transport der Blöcke in den Randbereich des Endinger Bruchs kommt nur die direkte Wirkung von fließendem Wasser in Frage. Weder Hangprozesse noch Eisschollendrift stellen aufgrund der topographischen Verhältnisse alternative Interpretationen dar. Fünf Schichtrichtungsmessungen in den kiesigen Sanden, eine höhere Anzahl konnte aufgrund der Aufschlußsituation nicht erbracht werden, zeigen eine Schüttungsrichtung nach Südwesten bis Westen (Einfallsrichtung 238 - 284 Einfallswinkel $5-29^{\circ}$ ). Es ergibt sich eine Übereinstimmung mit der rezenten Fließrichtung der ca. $1000 \mathrm{~m}$ entfernt gelegenen Barthe. Als kritische Erosionsgeschwindigkeit für einen angenommenen Korndurchmesser von $50 \mathrm{~cm}$ kann aus dem HJulström-Diagramm (in TuCKER 1985: 25) eine Fließgeschwindigkeit von ca. 5 - $9 \mathrm{~m} / \mathrm{s}$ entnommen werden. Auch wenn es sich am Fundplatz VI wahrscheinlich um eine „Schlamm- 
stromsedimentation" mit einem entsprechend geringeren Energieaufwand zum Transport der Blöcke gehandelt hat, sind Fließgeschwindigkeiten ähnlicher Dimension in der Barthe und ihren Nebenbächen nur unter kaltzeitlichen Bedingungen vorstellbar. Erklärbar ist der Transport der Steine und Blöcke sowie der kiesigen Sande durch zwei, eventuell kombinierbare Hypothesen:

1. Ereignishaft gelangte eine enorm hohe Wassermenge (z. B. zur Schneeschmelze im Frühjahr) während der Jüngeren Dryas aufgrund eines frostversiegelten Untergrundes (Permafrost oder lange jährliche Bodengefrornis) zum Abfluß und damit in das Becken.

2. Die Wassermenge wurde durch eine plötzliche natürliche Beckenentleerung oberhalb des Endinger Bruchs - im Sinne einer katastrophalen Ausflußvertiefung oder eines „Dammbruchs“ bereitgestellt. Die topographischen Voraussetzungen dafür waren bartheaufwärts aufgrund mehrerer höhergelegener Becken gegeben. Insgesamt deutet die Sedimenstruktur und -textur der Deckschichten am Fundplatz VI, wie auch in anderen Profilen vom Rand des Endinger Bruchs, auf ein fluviales Maximalereignis in der Jüngeren Dryas.

\section{Vegetationsrekonstruktion}

Methodische Vorbemerkungen

Nach Janssen (1966) sind die Zunahme der Entfernung von einer Pollenquelle lokaler, extralokaler und regionaler Pollenniederschlag zu unterscheiben. So lassen sich Vegetationsmuster durch Analyse mehrerer Kerne mit unterschiedlicher Distanz zu den Pollenquellen rekonstruieren (vgl. Janssen \& Braber 1987, De Klerk et al. 1997). Das Diagramm „Fundplatz VI, Sondierung 1 ( VI/S1)“ spiegelt den lokalen und extralokalen Pollenniederschlag an der Fundstelle wider (Abb. 6). Das ca. $1000 \mathrm{~m}$ entfernt gewonnene Diagramm „Hoher Birkengraben (HBG)“ aus dem Zentralteil des Endinger Bruchs reflektiert hingegen den regionalen Pollenniederschlag (Abb. 7). Die Pollenproben wurden mit $\mathrm{HCI}$ aufgearbeitet, in $\mathrm{KOH}$ (20\%) gekocht, gesiebt $(120 \mu \mathrm{m})$ sowie mit HF behandelt und acetolisiert (vgl. FAEGRI \& IVERSEN 1989). Die Diagramme beziehen sich auf eine Pollensumme von Pollentypen terrestrischer Pflanzen (linke Diagrammhälfte). Pollentypen, die möglicherweise auf Pflanzen von feuchten und nassen Standorten zurückgehen (rechte Diagrammhälfte), wie z. B. der Poaceae- und Cyper-
aceae-Typ, wurden ausgeschlossen, da die aufgrund (extra-)lokaler Überrepräsentation irreführend eine offene Landschaft suggerieren können (vgl. Janssen 1966, Janssen \& Ijzermans-LutGERHORST 1973). Das Verhältnis zwischen Baumpollen (AP) und terrestrischen Kräuterpollen (NAP) zeigt die relative Offenheit der Landschaft, die Spalte „Summe“ gibt die Höhe der Pollensumme wieder. Jede Pollenkurve wird mit dem tatsächlichen Prozentsatz (schwarz) sowie dem fünffach überhöhten Wert (offene Kurve mit Tiefenlinie der analysierten Proben) wiedergegeben. Die Pollentyp-Nomenklatur bezieht sich auf die morphologischen Beschreibungen von Moore et al. (1991) (M) und Faegri (1993) (F). Daneben wurde der Betula nana-Typ (*) mit kleinem Vestibulum vom Betula pubescens/undiff.-Typ (*) mit großem bzw. nicht deutlich sichtbarem Vestibulum getrennt.

Die Pollenkurven sind stratigraphisch geordnet, um die Vegetationssukzession klar hervortreten zu lassen. Auf Grund von Änderungen in der Pollenzusammensetzung sind die Diagramme in verschiedene biostratigraphische Zonen gegliedert, die nur der Vereinfachung der Diagrammbeschreibung dienen. Eine Korrelation beider Diagramme mit den Gliederungssystemen Mittelund Westeuropas führt zu Problemen, da diese nicht kompatibel sind (Bölling/Meiendorf-Problem; vgl. Bock et al. 1985, Hoek 1997, Usinger 1985). Aus diesem Grund wird die Korrelationstabelle durch einen Entwurf zur stratigraphischen Neugliederung des Spätglazials ergänzt (Abb. 8). Im folgenden werden nur die Ergebnisse zu Fundplatz VI vorgestellt. Eine spätere Studie wird ausführlicher die Entwicklung und räumliche Verbreitung der spätglazialen Vegetation im Endinger Bruch behandeln.

\section{Ergebnisse}

In beiden Diagrammen sind der Artemisia-, Helianthemum- und Chenopodiaceae-Typ sowie die Vaccinium-Sammelgruppe die wichtigsten NAPVertreter. Da die drei letzten Typen im Diagramm HBG mit höheren Werten auftreten als in VI/S1, ist davon auszugehen, daß Produzenten von diesen Pollentypen regional im Endinger Bruch eine wichtigere Rolle gespielt haben als lokal am Fundplatz VI. Sporen vom Selaginella-Typ - einem weiteren typischen Vertreter des Spätglazials - sind dagegen im Diagramm VI/S1 mit höheren Werten repräsentiert als in HBG, was auf eine extralokale Anwesenheit von Produzenten dieses Typs hinweist. 

an einem spätglazialen Fundplatz in Vorpommern

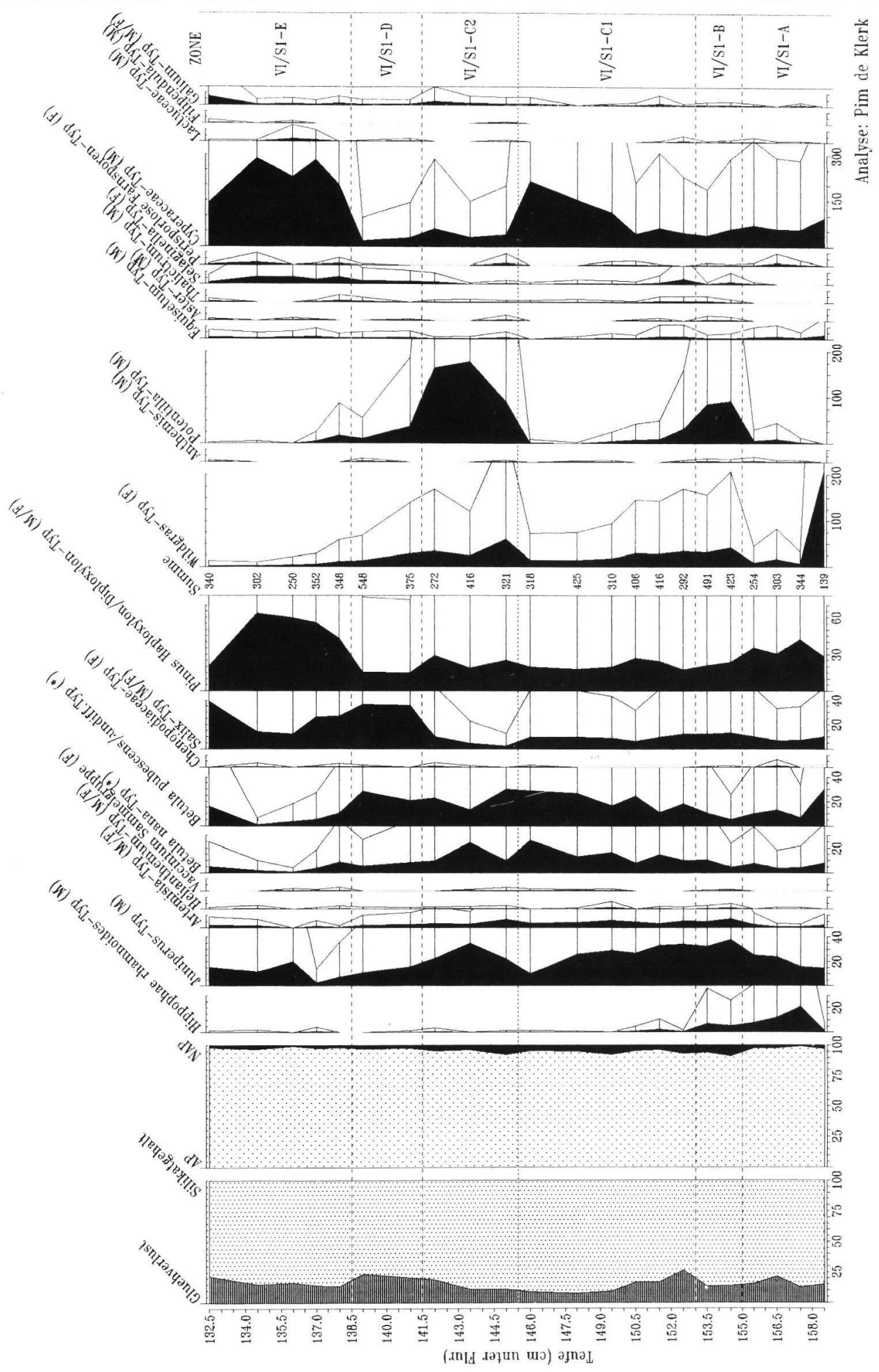

Abb. 6: Pollendiagramm Fundplatz Endingen VI, Sondierung 1 (VI S1), 124-151 cm, ausgewählte Pollenkurven.

Fig. 6: Pollendiagram site Endingen VI, exploration pitt (VI/S1), 124-151 cm, selected pollen-curves. 


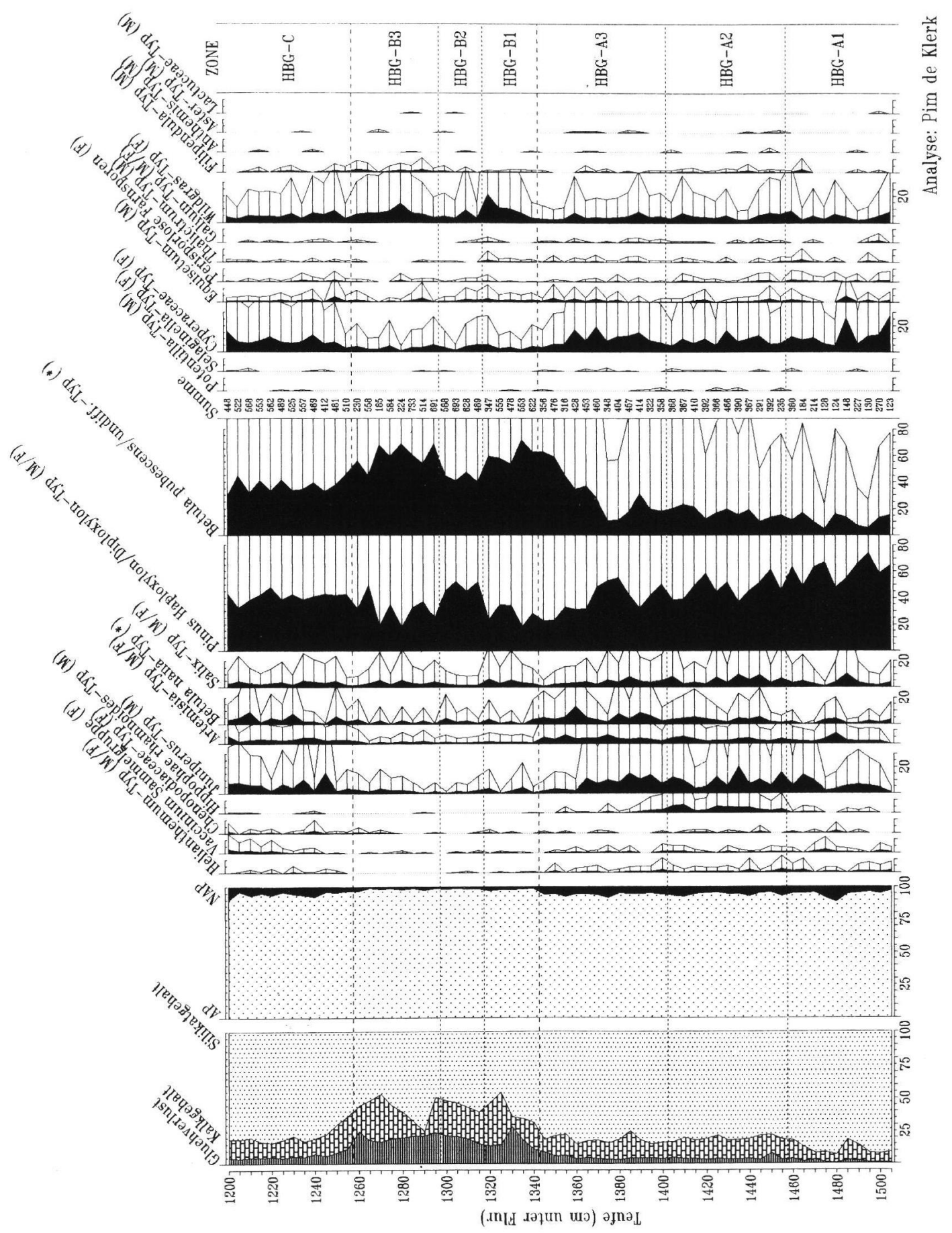

Abb. 7: Pollendiagramm Hoher Birkengraben (HBG), unterer Diagrammabschnitt, ausgewählte Pollenkurven.

Fig. 7: Pollendiagram Hoher Birkengraben (HBG), lower part, selected pollen-curves. 


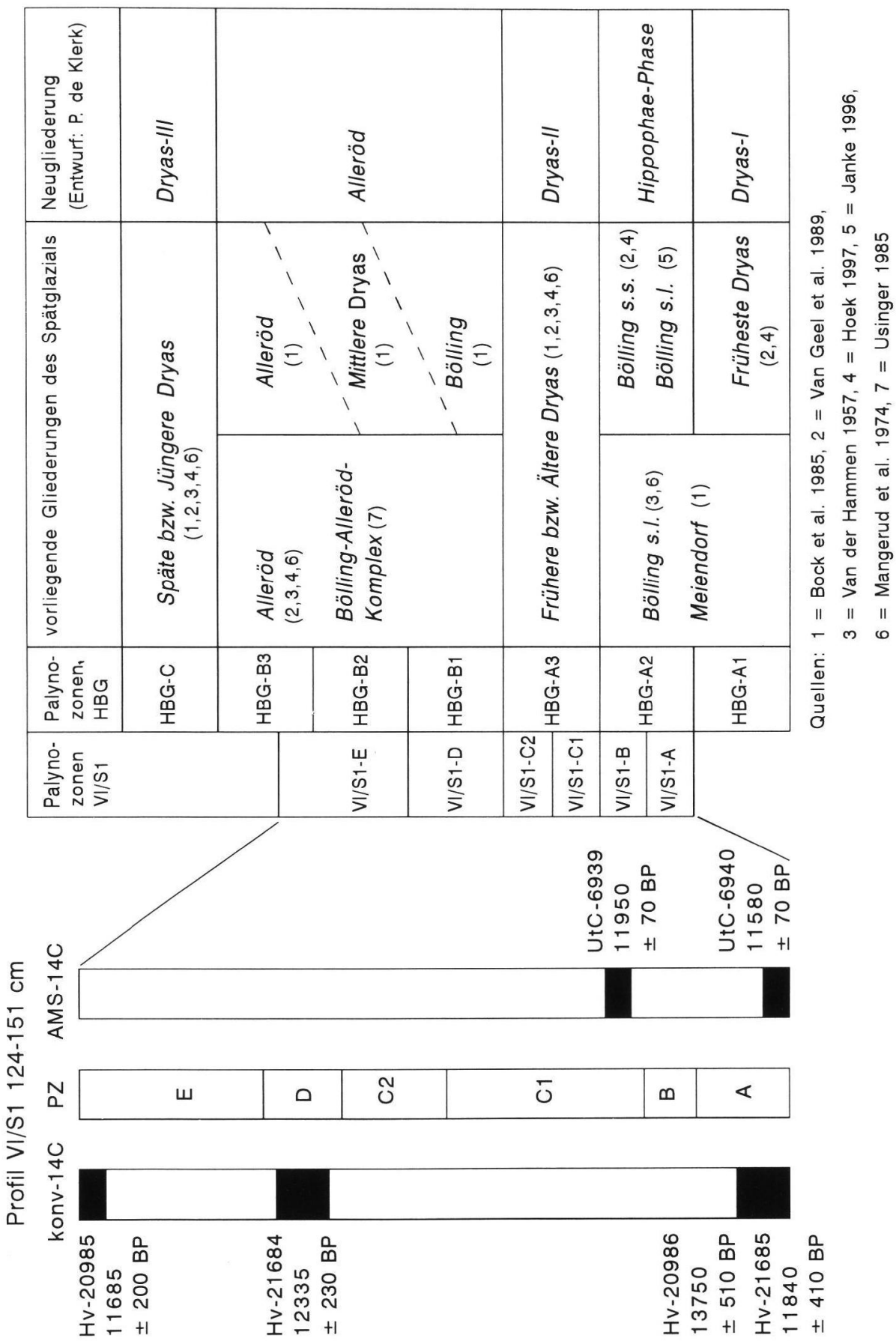

Abb. 8: Radiokarbondatierungen und Palynozonen der Organo-Silikatmudde in Profil VI/S1 (links) sowie Korrelation der Pollendiagramme VI/S1 und HBG mit stratigraphischen Gliederungen des Spätglazials (rechts).

Fig. 8: Radiocarbon dates and palynozones of the organic-silty mud of profile VI/S1 (left) and correlation of pollendiagrams VI/S1 and HBG with stratigraphical concepts of the Lateglacial (right). 
Ein gehäuftes Vorkommen des Hippophaë rhamnoides-Typs beschränkt sich auf den unteren $\mathrm{Ab}$ schnitt der Diagramme (Zonen HBG-A2, VI/S1-A und VI/S1-B). Eine ähnliche Phase tritt in vielen nordmitteleuropäischen Pollendiagrammen auf. Aus diesem Grunde schlagen wir vor, diesen Abschnitt als „Hippophaë-Phase“ zu bezeichnen. Ungehindert von konkurrierenden Arten kann Hippophaё sich in großen Beständen ausbreiten und ein baumähnliches Wachstum annehmen (Skogen 1972, Bokelmann et al. 1983). Ein Vergleich der NAP-Werte beider Diagramme zeigt, daß, obwohl regional im Endinger Bruch viele offene Stellen existieren, am Fundplatz VI die Vegetation ziemlich geschlossen war. Daher ist ein geschlossener Hipppophaë-Bestand anzunehmen. Der Rückgang von Hippophaë zu Beginn der Älteren Dryas (Dryas-II) kann möglicherweise mit einer Klimaverschlechterung in Verbindung gebracht werden, da der Sanddorn eine relativ wärmeliebende Pflanze ist (Kolstrup 1979). Die sehr hohen (extra-)lokalen Werte vom Juniperus-Typ, sowie die Zunahme der NAP-Pollen in den Zonen VI/S1-B, VI/S1-C1 und VI/S1-C2 zeugen von einer Ablösung des Hippophaë-Dickichts durch eine recht offene Juniperus-Strauchvegetation.

Am Ende der Älteren Dryas (HBG-A3, VI/S1-C2) ist in Profil HBG eine Abnahme des JuniperusTyps zugunsten des Betula nana-Typs (produziert von $B$. nana, B. humillis und möglicherweise von Hybriden zwischen verschiedenen BetulaArten) zu konstatieren, was auf eine regionale Ablösung des Wacholders durch die genannten Betula-Arten hinweist. Lokal am Fundplatz VI blieb Juniperus jedoch eine wichtige Komponente der Vegetation.

Auf den feuchten Standorten am Fundplatz haben Cyperaceae durchgehend eine dominierende Rolle gespielt (Wert ca. 50 - $75 \%$, HBG: 5 - 15\%), mit einem Maximum am Ende der Zone VI/S1-C1 (200\%). In Zone VI/S1-C2 wird der CyperaceaeTyp von Pollen des Potentilla-Typs abgelöst, die aufgrund von Samenfunden wahrscheinlich auf Potentilla palustre zurückgehen.

Die Pollenwerte von Betula pubescens-/undiff.Typ in Diagramm HBG sind ein Anzeichen dafür, daß in der ersten Phase des Alleröds (Zone HBGB1) Baumbirken eine wichtige regionale Vegetationskomponente bildeten. Am Fundplatz VI (Zone VI/S1-D) beherrschten jedoch Salix-Arten wahrscheinlich Strauchweiden - die Vegetation. $\mathrm{Zu}$ dieser Phase dürften die archäologischen Funde gehören.
Während der zweiten Phase des Alleröd (Zone VI/S1-E, HBG-2) ist Pinus am Fundplatz zu vermuten, deren Häufigkeit aufgrund der großen Pollenproduktion und der guten Pollenverbreitungsmöglichkeiten zu relativieren ist; auch Juniperus muß noch vorhanden gewesen sein. Die feuchteren Standorte wurden wiederum von Cyperaceae geprägt. Die letzte Phase des Alleröd (Zone HBG-B3) ist in Diagramm VI/S1 aufgrund einer Erosion dieser Sedimente in der Jüngeren Dryas (Dryas-III) nicht mehr vertreten.

\section{Radiokarbondaten}

Zum Fundplatz VI liegen zehn Radiokarbondatierungen vor (Tab. 1). Nur fünf Daten lassen sich nach der Konfrontation mit palynologischen, stratigraphischen und archäologischen Argumenten als plausibel bzw. genau genug bezeichnen. Insbesondere die konventionellen Datierungen an Gesamtsedimentproben der Organo-Silikatmudde von Profil VI/S1 lieferten sehr weite Vertrauensintervalle ( \pm 200 bis 510 Jahre) und fielen tendenziell zu alt aus. Letzteres könnte auf eine Kontamination der Mudden mit älterem Kohlenstoff zurückgehen („Reservoir-Effekt“", vgl. GEYH 1983: 31ff.). Auch zwei der insgesamt fünf AMS-14CDatierungen ergaben unbrauchbare, in diesem Falle zu junge Werte. Dafür könnten die sehr geringe Proben- und damit Kohlenstoffmenge des Makrorestes von Probe UtC-6940, die mögliche Datierung eines jüngeren Wurzelrestes bei Probe UtC-6973 sowie die mehrmonatige Lagerung der feuchten Proben verantwortlich sein (vgl. Wohlfahrth et al. 1998). Der Versuch, ein absolutes Alter des Beginns der spätglazialen Sedimentation im Profil VI/S1 zu erhalten, mißglückte aufgrund einer Datenreihe von 11580 $\pm 70 \mathrm{BP}, 11840 \pm 410 \mathrm{BP}$ und $13750 \pm 510 \mathrm{BP}$ aus einem Entnahmeniveau (Abb. 5, 8). Zwei termini ante quem der Hippophaë-Phase mit $11950 \pm 70$ BP (Profil VI/S1) und $11930 \pm 70$ BP (UtC-6935 aus Profil III-25, ca. 900 m von Fundplatz VI) lassen aber den Sedimentationsbeginn zwischen 12500 und $12000 \mathrm{BP}$ vermuten. Die Plausibilität der an den archäologischen Funden gewonnenen Daten wird im folgenden Abschnitt diskutiert.

\section{Archäologische Ergebnisse}

Mit der Wiederaufnahme der Geländearbeiten ist zwar die Lokalisierung des Fundplatzes, der in den Ortsakten unter der Bezeichnung Schuenhagen Fpl. 1 geführt wird, überzeugend gelungen, die spätglaziale Fundschicht konnte jedoch, trotz zahlreicher Sondagen nicht (mehr) aufgenom- 
Tab. 1: Radiokarbondaten vom Fundplatz Endingen VI. Kalibration mit 2-D Dipersion Calibration Program Version Cologne, Wenninger 1993.

Tab. 1: Radiocarbon dates of site Endingen VI. Calibrated by 2-D Dipersion Calibration Program Version Cologne, Wenninger 1993.

\begin{tabular}{|l|l|l|l|l|l|l|l|l|}
\hline Nr. & Profil & Tiefe $(\mathbf{c m})$ & Datum BP & Datum cal BC & Labor-Nr. & Methode & Material & $\begin{array}{l}\text { Datum } \\
\text { plausibel? }\end{array}$ \\
\hline 1 & VI/S1 & $131,5-132,5$ & $11685 \pm 200$ & $11665 \pm 224$ & Hv-20985 & konv & Organo-Silikatmudde & ja \\
\hline 2 & VI/S1 & $139,0-141,0$ & $12335 \pm 230$ & $12468 \pm 312$ & Hv-21684 & konv & Organo-Silikatmudde & nein \\
\hline 3 & VI/S1 & $151,5-152,5$ & $11950 \pm 70$ & $11977 \pm 84$ & UtC-6939 & AMS & Makrorest von Carex & ja \\
\hline 4 & VI/S1 & $156,5-158,5$ & $13750 \pm 510$ & $14475 \pm 656$ & Hv-20986 & konv & Organo-Silikatmudde & nein \\
\hline 5 & VI/S1 & $156,5-158,5$ & $11840 \pm 410$ & $11854 \pm 484$ & Hv-21685 & konv & Organo-Silikatmudde & nein \\
\hline 6 & VI/S1 & $157,5-158,5$ & $11580 \pm 70$ & $11551 \pm 78$ & UtC-6940 & AMS & Periderm-Material & nein \\
\hline 7 & VI/S4 & $153,5-155,5$ & $12360 \pm 245$ & $12506 \pm 366$ & Hv-20987 & konv & Makrorest von Salix & ja \\
\hline 8 & VI/S4 & $160,0-161,0$ & $9700 \pm 200$ & $8773 \pm 316$ & UtC-6973 & AMS & Makrorest von Carex & nein \\
\hline 9 & Altfund & Riesenhirsch & $11555 \pm 100$ & $11522 \pm 110$ & UZ-3798 & AMS & Geweih Riesenhirsch & ja \\
\hline 10 & Altfund & Pferd & $11830 \pm 50$ & $11835 \pm 60$ & UtC-5681 & AMS & Rippe Pferd \\
\hline
\end{tabular}

men werden. Eine angeschnittene Strate des späten Frühmesolithikums wurde kleinräumig freigelegt. Die geowissenschaftlichen Arbeiten haben für das Alleröd ein Stillgewässer nachgewiesen, aus dessen muddigen Sedimenten nach Deecke (1900a, b) die Faunenreste geborgen wurden. Der eigentliche Lagerplatz hat am Ufer dieses Gewässers gelegen. Schnell fließendes Wasser führte in der Jüngeren Dryas wahrscheinlich zu einer Ausräumung der höhergelegenen Sedimente, so daß vom ursprünglichen Lagerplatz weder Befunde noch Funde überliefert sind. Das vorhandene Material kann demnach als eine im Wasser gelegene Abfallzone aufgefaßt werden. Diese Fundumstände scheinen mit dafür verantwortlich, daß ausschließlich Faunenreste geborgen wurden und keine Steinartefakte. Das Fundmaterial, das überwiegend in der Geologischen Sammlung der Universität Greifswald aufbewahrt wird, besteht aus 44 Fragmenten, die zu 40 Geweih- und Knochenresten gehören (STREET 1996, Terberger 1996a). Obwohl der Kontext und das Ausmaß der Fundstreuung nur vage bekannt ist, läßt Homogenität des Materials an ein ursprünglich relativ begrenztes Fundinventar denken: Allein der Elch konnte mit acht, z. T. mit Schnittund Schlagspuren versehenen Knochenfragmenten als eindeutige Jagdbeute identifiziert werden (Abb. 9, 10). 22 Knochenfragmente sind als Reste von mindestens zwei großen Cerviden anzusprechen; diese gehören vermutlich ebenfalls zu Elch. Mit vereinzelten Fragmenten ist auch Hecht (zweimal) und Ente (einmal) vertreten. Hervorzuheben sind eine messerartig angeschärfte Pferderippe sowie eine als „Kern“ genutzte Abwurfstange vom Riesenhirsch (Abb. 10). Beide Objekte sind als Werkzeug bzw. Werkstück wahrschein- lich mit nach Endingen gebracht worden, zumal sie die Spezies jeweils als Einzelstücke vertreten. Ähnlich zugerichtete „Rippenmesser" vom Pferd sind aus Fundschichten der Rentierjägerstationen Meiendorf und Stellmoor bekannt, die in den Kontext der Hamburger Kultur gehören. Die „Rippenmesser" können jedoch nicht als exklusive Form des Spätglazials gewertet werden, da z. B. ein ganz ähnliches Werkzeug aus slawischem Zusammenhang bekannt geworden ist. Bisher ohne Parallelen im Spätglazial steht die Nutzung eines Riesenhirschgeweihs für die Gewinnung von Grundformen. An dem Geweihfragment lassen sich die typischen Merkmale der Spangewinnungstechnik ablesen (Abb. 10): Begradigte Kanten und zwei stehengebliebene Enden herausgetrennter Späne erlauben den fortgeschrittenen Abbau des Geweihs nachzuvollziehen. Die Späne sind als Rohformen für Geschoßspitzen (mit Widerhaken) anzusehen (Terberger 1997: 18, STREET \& BAAles 1997: 379). In einer folgenden Nutzungsphase des Geweihstückes wurde die Spongiosa entfernt, der Zweck dieser Bearbeitung ist unklar. Das Geweihstück ist nicht nur ein seltener Nachweis des Riesenhirsches in Norddeutschland, sondern auch der Spangewinnungstechnik im Alleröd. Der Fund ist damit auch ein Bindeglied zwischen spätglazialer und holozäner (mesolithischer) Technologie.

Die Datierung der Funde in das Alleröd-Interstadial ergibt sich aus stratigraphischen Überlegungen, die mit zwei AMS-14C-Daten eine Bestätigung finden: Ein Datum von $11830 \pm 50$ BP (UtC5681) für die angeschärfte Pferderippe stellt das älteste ${ }^{14} \mathrm{C}$-Datum aus archäologischem Kontext für Mecklenburg-Vorpommern dar. Das Datum für das Riesenhirschgeweih fällt mit $11555 \pm 100$ 
nachgewiesene Jagdbeute

nachgewiesene Fauna (Jagdbeute?)

Knochenwerkzeug bzw. Geweih-Werkstück
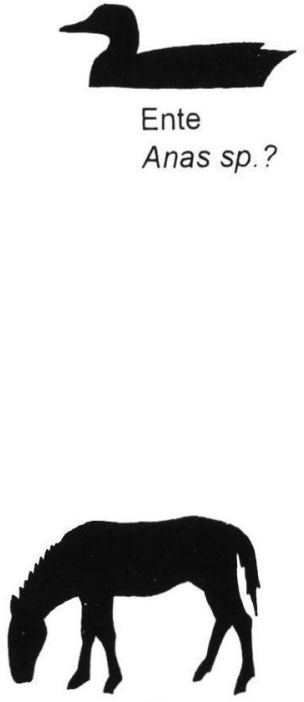

Pferd

Equus $s p$

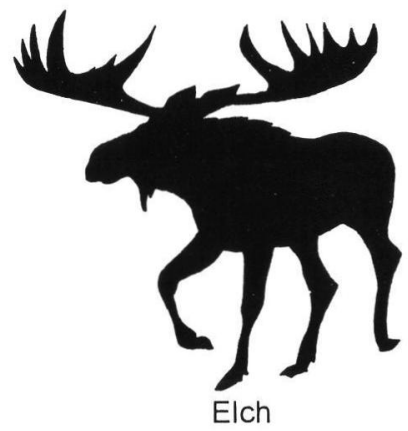

Alces alces

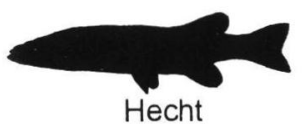

Esox lucius

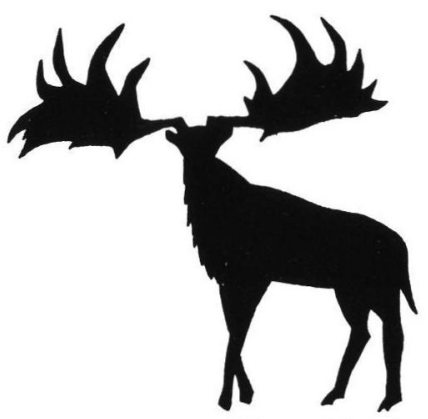

Riesenhirsch

Megaloceros giganteus

Abb. 9: Spätglaziale Fauna am Fundplatz Endingen VI.

Fig. 9: Lateglacial fauna of site Endingen VI.

BP (UZ-3798) deutlich jünger aus. Für die Interpretation der Daten kommt eine Teilung der Funde in zwei Fundschichten in Betracht. Dann würde die Pferderippe zu einer älteren Belegungsphase gehören. Allerdings ist auch eine zeitliche Streuung von Daten für einen Horizont und damit ein methodisches Problem möglich. Größere $\mathrm{Da}-$ tierungsabweichungen eines Ereignisses mögen AMS-14C-Daten an Knochenresten aus der Grotte du Bichon, Schweiz, beispielhaft illustieren: Zwei Daten an einem Bärenskelett ergaben eine Differenz von 320 Jahren (ETH-8301: $11680 \pm 90 \mathrm{BP}$, ETH-8775: $11360 \pm 120$ BP). Bezieht man zwei weitere Datierungen ein, die an einem dazugehörigen, menschlichen SKelett ermittelt wurden, erhöht sich der Datierungsbereich für diesen Jagdunfall, bei dem Mensch und Bär zur Tode kamen, sogar auf 400 Jahre (MOREL 1993). In Endingen möchte man das Gros der Funde zu einer Aufenthaltsphase rechnen, was eine Einstreuung älterer Elemente nicht ausschließt. Beide Daten ordnen die Fundschicht(en) in das frühe Alleröd ein. Zusammenfassend kann der Fundplatz als Elchjägerplatz angesprochen werden. Ein reiner Schlachtplatz ist aufgrund des Rippenmessers und des Geweihkerns unwahrscheinlich. Letzte- 

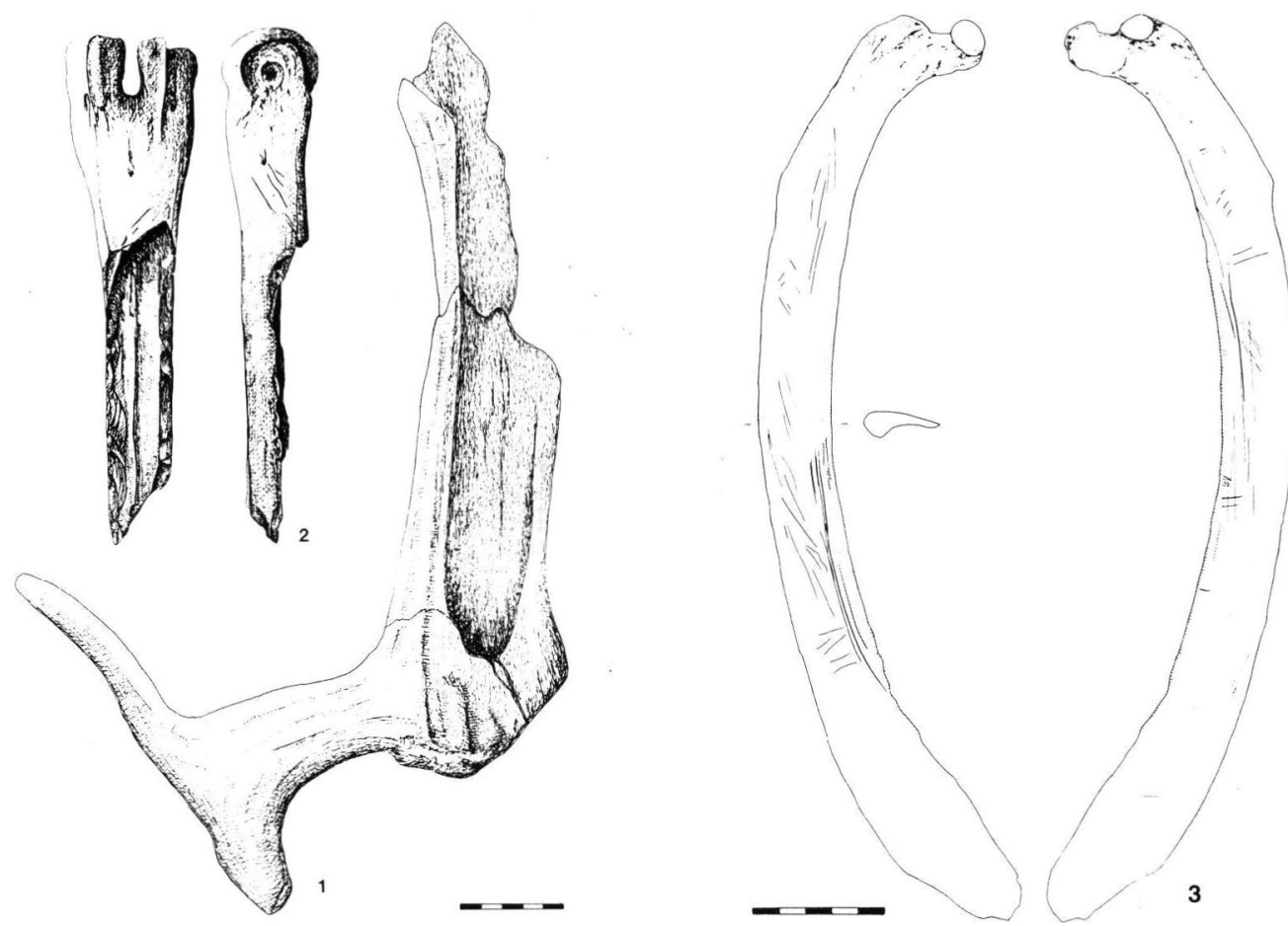

Abb. 10: Archäologische Funde vom Fundplatz Endingen VI. 1 Riesenhirschgeweih mit Spuren der Spangewinnung, 2 angeschärfte Pferderippe, 3 Elchknochen mit Schlagmarken und Schnittspuren.

Fig. 10: Archaeological finds of site Endingen VI. 1 Antler of giant deer with traces of groove and splinter tech nique, 2 sharpened horse-ribs, 3 elk-bone with blow-marks and cut-marks.

rer weist auf die Fertigung von Geschoßspitzen am Ort hin. Die angeschärfte Rippe mag zum Schneiden von Pflanzen gedient haben, aber auch z. B. eine Funktion bei der Fellverarbeitung kommt in Betracht. Das Fehlen von Zeugnissen der Feuersteinbearbeitung ist wohl auf die Fundsituation und die Bergungsumstände zurückzuführen. Das kleine Endinger Ensemble erhält seine Bedeutung durch die Seltenheit von Fundstellen des Spätglazials mit erhaltenen Faunenresten. Gemeinsam mit dem Fundplatz Lüdersdorf, Kr. Nordwestmecklenburg, repräsentiert er einen seltenen Beleg für die Jagd bzw. Nutzung des Riesenhirsches im Spätglazial (BRATLund 1993, OxA3615: $11600 \pm 105$ BP). Mit dem Elch als nachgewiesene Jagdbeute entspricht der Fundplatz Endingen VI dem - allerdings recht lückenhaften Bild der allerödzeitlichen Fundstellen Norddeutschlands und Südskandinaviens (ERIKSON 1996: 13, 19). Im Rheinland scheint im Alleröd demgegenüber der Rothirsch als Jagdbeute im Vordergrund zu stehen und der Elch zusammen mit Reh und Ur erst in zweiter Linie gejagt worden zu sein (STREET \& BAaLEs 1997: 376).

\section{Megaloceros giganteus im Spätglazial des nördlichen Mitteleuropa}

Der 1899 geborgene Geweihrest des Riesenhirsches (Megaloceros giganteus BlumENBACH, 1803) ist der Anlaß, einen näheren Blick auf diese Art im Spätglazial des nördlichen Mitteleuropas zu richten. Seine Körpergröße (Schulterhöhe bis 1, $8 \mathrm{~m}$ ) und das ausladende Geweih, das nach einem irischen Fund von Cheswardine eine Auslage von bis zu $369 \mathrm{~cm}$ erreichte (STUART 1991: 503; KAHLKE 1994: $35 \mathrm{ff}$ ), reiht Megaloceros in die Gruppe der imposanten spätglazialen Großsäuger ein.

Jungpaläolithische Höhlenmalereien und -gravierungen bezeugen, daß auch der eiszeitliche Mensch von diesem großen Cerviden beeindruckt war. Im südwest-französischen Departement Quercy ist der Riesenhirsch mit 20 Darstellungen in drei Höhlen gehäuft zu finden, während er im Perigord mit einem Beispiel vertreten ist (LORBLANCHET 1997: 58). In der Höhle von Cougnac nehmen drei Riesenhirsche eine markante Stellung innerhalb des Darstellungsensembles ein (LORBLANCHET 1984: 486). Drei Direkt- 
datierungen der Farbreste stellen diese Malereien in das (jüngere) Gravettien (25120 - $22750 \mathrm{BP})$. Ein jüngeres Datum von $19500 \pm 270 \mathrm{BP}$ scheint auf eine spätere Ausgestaltungsphase zu verweisen, in der die Tiere vermutlich teilweise übermalt wurden (LORBLANCHET 1997: 268). Der Riesenhirsch ist für das Eem und das letzte Glazial im gesamteuropäischen Raum belegt (STUART 1991, Koenigswald \& Heinrich 1996, Koenigswald \& Rosendahl 1997). Für das Spätglazial nimmt die irische Fundstelle Ballybetagh mit mehr als 100 Schädelfunden des Riesenhirsches eine besondere Stellung ein. Diese werden in das dem Alleröd entsprechende Woodgrange-Interstadial datiert. Im nördlichen Mitteleuropa lassen sich eine Reihe von datierten Nachweisen von Megaloceros giganteus aus dem Weichselspätglazial anführen (Abb. 11). Zwei Funde aus Schlutup bei Lübeck wurden durch GUENTHER (1955) aufgrund lithostratigraphischer Gesichtspunkte in ein Interstadial zwischen Pommerschem und Mecklenburger Stadium gestellt („Lockarp-Interstadial“, vgl. DuPHORN et al. 1995: 137). Chronologisch schließst sich eine Reihe von sechs radiokarbondatierten Riesenhirschfunden aus Norddeutschland, Dänemark und Südschweden an. Diese belegen ein allerödzeitliches Vorkommen der Art, das eventuell bis in die Jüngere Dryas hineinreicht. Mit einer zeitlichen Lücke folgt ein Riesenhirschfund von Theresienhof bei Plön. Er soll nach lithostratigraphischen und pollenanalytischen Untersuchungen in den Übergang Jüngere Dryas/Präboreal oder in das Präboreal datieren (GuENTHER 1960, TIDELSKI 1960). Zwar ist die genannte pollenanalytische Untersuchung nicht in Zweifel zu ziehen, die Bergungsumstände lassen jedoch die wünschenswerte Eindeutigkeit vermissen: Das Stück wurde bei Baggerungen zufällig entdeckt und erst später aufgrund von anhaftenden Sedimentresten einem Horizont zugewiesen. Da dieser gegenüber den absolut datierten Exemplaren deutlich jünger eingestufte Riesenhirschfund isoliert steht, wäre eine Überprüfung wünschenswert.

Zusammenfassend ist festzuhalten, daß die bislang 21 Funde aus Norddeutschland (5), Dänemark (10) und Südschweden (6) in der Regel nicht jünger als Alleröd datieren. Sollte sich die Datierung des Fundes von Plön in das beginnende Präboreal nicht bestätigen, so wäre der Riesenhirsch im nördlichen Mitteleuropa nach dem jetzigen Stand ab der frühen Jüngeren Dryas nicht mehr vertreten. In Sibirien lassen sich die jüngsten Reste von Megaloceros in der jungpaläolithischen Station Verhlenskaa Gora bei Irkutsk auf
12570 BP datieren (KAHLKE 1994: 35 ff). Die bisher jüngsten datierten Funde überhaupt stammen von Irland und könnten für ein Fortleben von $\mathrm{Me}$ galoceros in dieser Region bis in die Jüngere Dryas sprechen (Ballybetagh: $10610 \pm 495 \mathrm{BP}$; Kirkhead Cave: $10700 \pm 200$ BP, konv. Daten; STUART 1991: 504). Fünf neue irische AMS-14CDaten an spätglazialen Exemplaren von Megaloceros ergaben jedoch Daten zwischen $11820 \pm$ $120 \mathrm{BP}$ und $10960 \pm 110 \mathrm{BP}$ (WoODMAN et al. 1997). D. h. bei Berücksichtigung dieser Daten und der großen Vertrauensintervalle der älteren Daten läßt sich ein Vorkommen in der Jüngeren Dryas bislang nicht sicher belegen. Der Mensch als Jäger wird das Aussterben der Art nicht ausgelöst haben, vielmehr hat der Riesenhirsch als Jagdbeute nach jetzigem Kenntnisstand im Spätglazial kaum eine Rolle gespielt. Die Bejagung mag den natürlichen Prozeß des Aussterbens beschleunigt haben.

\section{Zusammenfassende Diskussion}

In Abb. 12 wird eine modellartige Zusammenfassung der geowissenschaftlichen und archäologischen Ergebnisse zum Fundplatz VI gezeigt. Die am Fundplatz VI aufgeschlossenen Seeablagerungen datieren nach Pollenanalysen und ${ }^{14} \mathrm{C}-\mathrm{Daten}$ zweifelsfrei in das Spätglazial. Ein Bezug ihrer jungdryaszeitlichen Deckschichten zu glazialen Vorschüttsanden des „Langeland-Vorstoßes“/der „Velgaster Staffel“ sensu Gross (1954) bzw. ein "Interglazial von Endingen" sensu WOLDSTEDT (1955) müssen daher abgelehnt werden. Die in einem Becken lokal nachgewiesene Abfolge von limnischen Sedimenten des älteren Spätglazials und fluvialen Sedimenten der Jüngeren Dryas fügt sich gut in das regionale Bild ähnlicher Sedimentationsräume ein (z. B. KLIEWE 1989, KAISER \& Terberger 1996, Kaiser \& Janke im Druck). Interessant ist ein aus der Uniformität der Mudden ableitbarer Hinweis auf lokale morphodynamische Stabilität zwischen der Hippophaë-Phase und dem Alleröd. Andere Profile aus dem Endinger Bruch in der Nähe heutiger Fließgewässer zeigen hingegen einen deutlichen fluvialen Einfluß während der Älteren Dryas: d. h. es können offensichtlich faziell bedingt bereits in einem Becken die stratigraphischen Aufzeichnungen kleinräumig erheblich voneinander abweichen. Dies muß bei der regional bislang üblichen Ausdeutung von Einzelprofilen und bei der Profilkorrelation über größere Entfernungen hinweg beachtet werden. Zu prüfen bleibt weiterhin, ob sich auch anderswo fluviale Maximalereignisse 


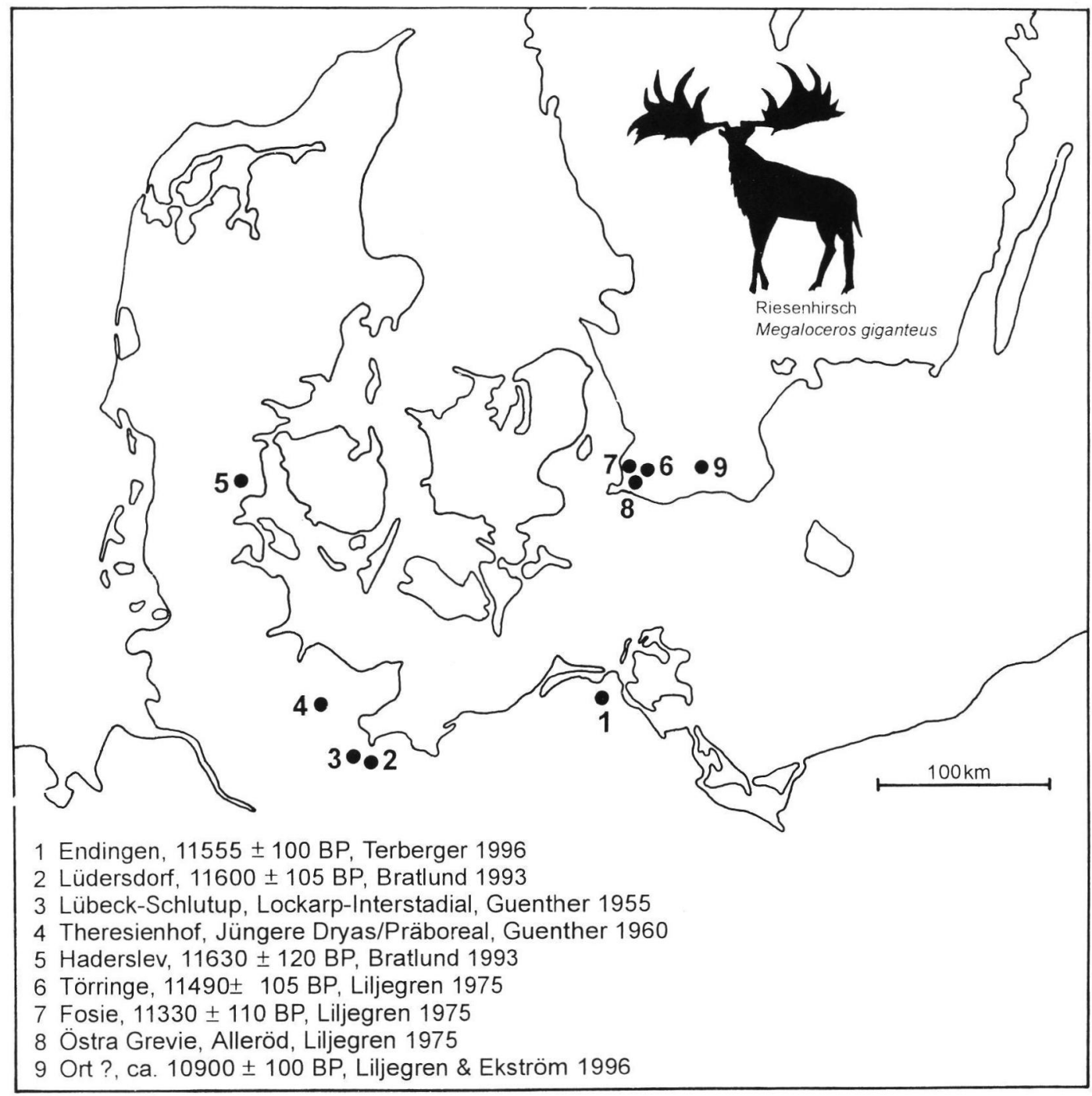

Abb. 11: Datierte Nachweise des Riesenhirsches (Megaloceros giganteus) aus dem Spätglazial des nördlichen Mitteleuropa.

Fig.: Dated occurences of giant deer (Megaloceros giganteus) in northern Central-Europe.

während der Jüngeren Dryas nachweisen lassen und welche Ursachen dafür in Betracht kommen. $\mathrm{Da}$ in ausgedehnten Beckenarealen, wie z. B. in der Rostocker Heide oder in der Ueckermünder Heide, ungegliederte Sande der Jüngeren Dryas nachgewiesen wurden, liegt die Vermutung nahe, daß auch dort wenige fluvial-limnische oder äolische Ereignisse die Sedimentation verursacht haben.

Das regionale Pollenbild im Diagramm HBG zeigt eine klassische spätglaziale Palynostratigraphie des nördlichen Mitteleuropas. Ein Vergleich des Diagramms HBG mit dem Diagramm VI/S1 illu- striert, daß am Fundplatz VI in allen Palynozonen lokal eine strauchreiche Vegetation existierte. Ein ausgeprägtes Maximum von Hippophaë zeigt sich in vielen nordmitteleuropäischen Diagrammen in einer ähnlichen biostratigraphischen Position.

Aus diesem Grunde wird vorgeschlagen, diese als „Hippophaë-Phase“ zu bezeichnen, um damit Abstand von der traditionellen, jedoch irreführenden Gliederungssystemen zu gewinnen (Abb. 8). Während der Älteren Dryas dominierten Juniperus-Sträucher, die mit Betula nana bzw. B. bumilis in geringerer Menge vergesellschaftet waren. Am Anfang des Alleröd prägten Salix-Sträu- 

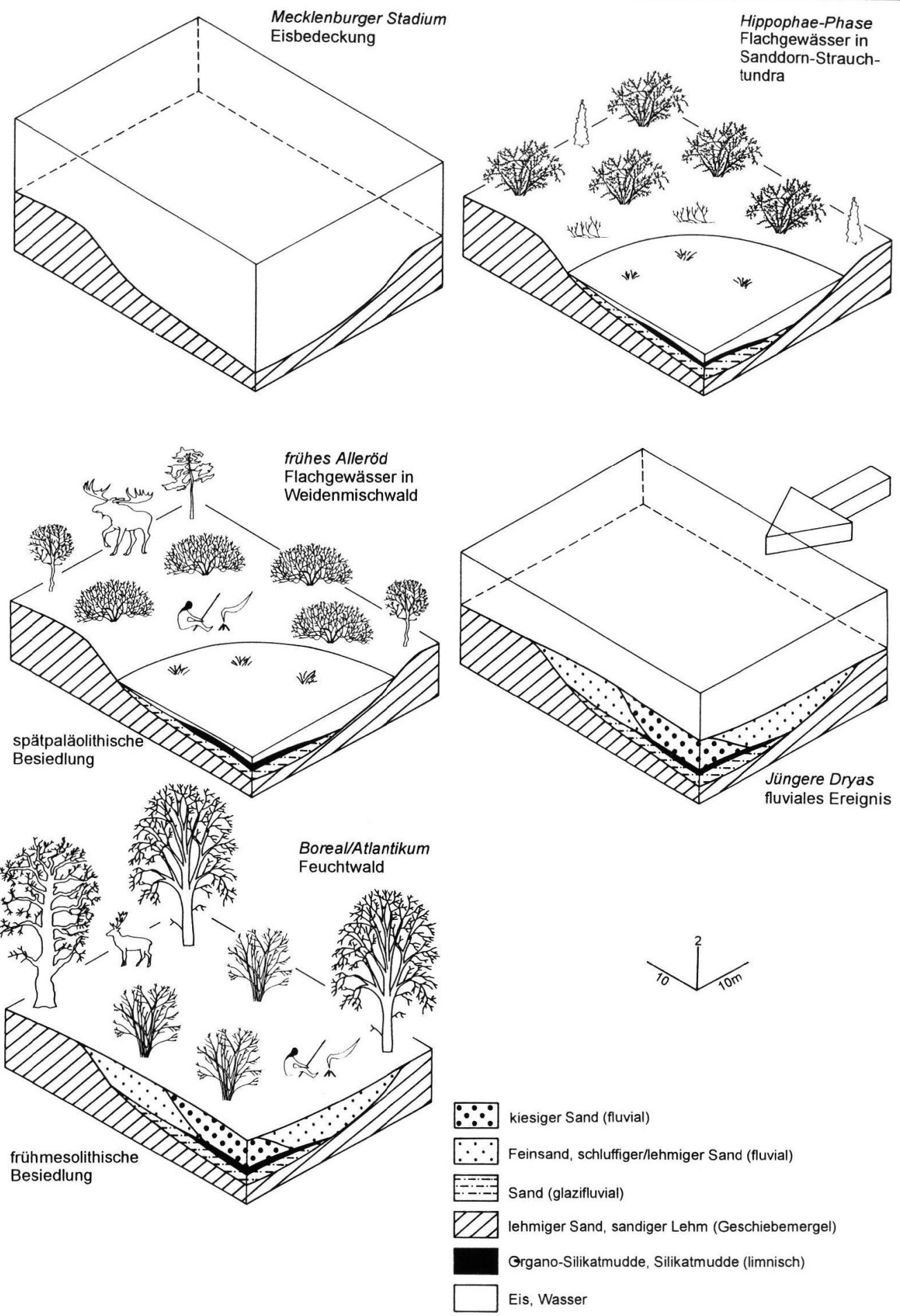

Abb. 12: Schematische Übersicht zur Landschaftsentwicklung am Fundplatz Endingen VI.

Fig. 12: Schematic model of landscape development at site Endingen VI. 
cher die Umgebung des Fundplatzes, während später auch Pinus aufkam.

Aus archäologischer Sicht repräsentiert der Elchjägerplatz Endingen den ältesten absolut datierten Nachweis einer menschlichen Besiedlung in Nordostdeutschland. Ein Zusammenhang des Fundplatzes mit den Federmessergruppen des Alleröd ist sehr wahrscheinlich, aufgrund des Fehlens von Steinartefakten unter den Funden aber nicht zu belegen. Spuren einer noch älteren Besiedlung durch die Rentierjäger der (späten) Hamburger Kultur lassen sich trotz solcher Nachweise im dänischen Jütland und auf Seeland für Mecklenburg-Vorpommern bisher nicht klar fassen (Terberger 1997). Berücksichtigt man die traditionellen typologischen Erkenntnisse, so lassen sich weitere Fundstellen der Federmessergruppen (Alleröd) sowie der Rentierjäger der Ahrensburger Kultur (Jüngere Dryas) inzwischen in gewachsener Zahl anführen (TERBERGER 1996b).

\section{Schriftenverzeichnis}

AG BODEN (1994): Bodenkundliche Kartieranleitung (4. Auflage ). - 392 S.; Hannover.

Billwitz, K., Helbig, H., Kaiser, K. \& Terberger, T. (1998): Geländebefunde zur spätglazialen Naturraumgenese und Besiedlungsgeschichte von Becken und Platten in Vorpommern. - Z. Geomorph. N. F., Suppl.-Bd. 112: 123 - 142; Stuttgart.

Bock, W., Menke, B., Streht, E. \& Ziemus, H. (1985): Neuere Funde des Weichselspätglazials in Schleswig-Holstein. - Eiszeitalter und Gegenwart, 35: 161 - 180; Öhrigen.

Bokelmann, K., Heinrich, D. \& Menke, B. (1983): Fundplätze des Spätglazials am Hainholz-Esinger Moor, Kreis Pinneberg. - Offa, 40: 199 - 239; Neumünster.

Bratlund, B. (1993): Ein Riesenhirschschädel mit Bearbeitungsspuren aus Lüdersdorf, Kreis Grevesmühlen. - Offa 49/50: 7-14; Neumünster.

Deecke, W. (1900a): Ueber ein Vorkommen von bearbeiteten Säugetierresten bei Endingen (Kreis Franzburg). - Festschrift zur Feier des 50. Doktorjubiläums von Geheimrat Limpricht: 1-10; Greifswald.

- (1900b): Vorkommen von bearbeiteten Riesenhirschknochen bei Endingen (Kreis Franzburg) in Vorpommern. - Globus, 78 (1): 13-15; Braunschweig.

Duphorn, K., Kliewe, H., Niedermeyer, R.-O., Janke, W. \& Werner, F. (1995): Die deutsche Ostseeküste. Sammlung geologischer Führer 88. - 281 S.; Berlin, Stuttgart.

FAEGRI, K. (1983): Bestimmungsschlüssel für die nordwest-europäische Pollenflora. - Auszug aus FAEGRI, K \& IverSEN, J. (1989), übersetzt von E. LANGe, 85 S.; Jena.
— \& Iversen, J. (1989): Textbook of Pollen analysis, 4th edn., (revised by Faegri, K. \& Kaland, P. E. \& KRZYWINSKI, K.) 328 S.; Chichester.

ERIKSON, B. V. (1996): Regional variation in Late Pleistocene subsistence strategies. Southern Scandinavian reindeer hunters in an European context. - In: LARSsON, L. (Hrsg.): The earliest settlement of Scandinavia and its relationship with neighbouring areas. Acta Archaeologica Lundensia ser. 8, 24: 7 - 22; Stockholm.

Van Geel, B., Coope, G. R. \& VAn Der Hammen, T. (1989): Palaeoecology and stratigraphy of the lateglacial type section at Usselo (The Netherlands). - Rev. Palaeobot. Palynol., 60: 25 - 129; Amsterdam.

GEOLOGISCHES LANDESAMT MECKLENBURG-VORPOMMERN (1995): Geologische Karte von Mecklenburg-Vorpommern. Karte der quartären Bildungen - Oberfläche bis fünf Meter Tiefe 1:200000. Blatt Bad Doberan/Rostock; Schwerin.

GeYh, M. (1983): Physikalische und chemische Datierungsmethoden in der Quartär-Forschung. - Clausthaler Tektonische Hefte 19, 163 S.; Clausthal-Zellerfeld.

Gross, H. (1938): Endingen Kr. Franzburg-Barth, Fundort von Riesenhirschresten. - Unveröff. Mskr., ErnstMoritz-Arndt-Universität Greifswald, Historisches Institut, Lehrstuhl f. Ur- und Frühgeschichte, o. S.; Greifswald.

- (1954): Das Alleröd-Interstadial als Leithorizont der letzten Vereisung in Europa und Amerika. - Eiszeitalter und Gegenwart, 4/5: 189-209; Öhringen.

- (1958): Die bisherigen Ergebnisse von ${ }^{14} \mathrm{C}-$ Messungen und paläontologischen Untersuchungen für die Gliederung und Chronologie des Jungpleistozäns in Mitteleuropa und den Nachbargebieten. - Eiszeitalter und Gegenwart, 9: 155-187; Öhringen.

Guenther, E.-W. (1955): Diluviale Großsäuger aus Schleswig-Holstein und ihre zeitliche Einordnung. Schriften Naturw. Ver. Schleswig-Holstein, 27 (2): 99 -112; Kiel

- (1960): Funde von Ren und Riesenhirsch in nacheiszeitlichen Schichten aus der Nähe von Theresienhof bei Plön (Trentmoor und Brennacker). Schriften Naturw. Ver. Schleswig-Holstein, 30: 79-91; Kiel

VAN DER HAMmen, T. (1957): The stratigraphy of the LateGlacial. - Geol. Mijnbouw, 19: 250 - 254; 's Gravenhage.

Hoek, W. Z. (1997): Palaeogeography of Lateglacial vegetations. Aspects of Lateglacial and Early Holocene vegetation, abiotic landscape, and climate in The Netherlands. - 147 S.; Utrecht (Koninklijk Nederlands Aardrijkskundig Genootschap: Nederlandse Geografische studies 230).

JANKE, W. (1996): Biostratigraphische Untersuchungen am spätpaläolithischen Fundplatz Nienhagen, Lkr. Nordvorpommern. - Bodendenkmalpflege in Mecklenburg-Vorpommern, Jahrbuch 1995: 49-56; Lübstorf. 
JANSSEN, C. R. (1966): Recent pollen spectra from the deciduous and coniferous-deciduous forest of northeastern Minnesota: a study in pollen dispersal. Ecology, 47: 804-88825; Durham.

- \& Braber, F. I. (1987): The present and past grassland vegetation of the Chajoux and Moselotte valleys (Vosges, France). 2. Dynamic aspects and origin of grassland vegetation in the Chajoux valley, interpreted from the contrast between regional and local pollen deposition of dominant pollen types and the distribution of pollen indicators in pollen diagrams. - Proc. Kon. Ned. Akad. van Wetensch. C, 90: 115-138; Amsterdam.

— \& Ijzermans-Lutgerhorst, W. (1973): A „local“ LateGlacial pollen diagram from Limburg, Netherlands. - Acta Bot. Neerl., 22: 213-220; Oxford.

KaHlKe, R. - D. (1994): Die Entstehungs-, Entwicklungsund Verbreitungsgeschichte der oberpleistozänen Mammuthus-Coelodonta-Faunenkomplexes in Eurasien (Großsäuger). - Abh. d. Senckenb. Naturf. Gesell. 546, 164 S.; Frankfurt a. M.

Kaiser, K. \& Terberger. T. (1996): Archäologisch-geowissenschafliche Untersuchungen am spätpaläolithischen Fundplatz Nienhagen, Lkr. Nordvorpommern. - Bodendenkmalpflege in Mecklenburg-Vorpommern 43, Jahrbuch 1995: 7 - 48; Lübstorf.

- \& JANKE, W. (in Druck): Bodenkundlich-geomorphologische und paläobotanische Untersuchungen im Ryckbecken bei Greifswald (Wackerow-Fundplatz 1). - Bodendenkmalpflege in MecklenburgVorpommern 45, Jahrbuch 1997; Lübstorf.

De Klerk, P., Janssen, C. R., Joosten, J. H. H. \& TörnQUIST, T. E. (1997): Species composition of an alluvial hardwood forest in the Dutch fluvial area under natural conditions (2700 cal year BP). - Acta Bot. Neerl., 46: 131-146; Oxford.

KuIEwE, H. (1989): Zur Entwicklung der Küstenlandschaft im Nordosten der DDR während des Weichsel-Spätglazials. - Acta Geographica Debrecina 24 / 25: 99-113; Debrecen

Koenigswald, W. von \& Heinrich, W. - D. (1996): Kurze Charakterisierung der Veränderungen in der Säugetierfauna des Jungwuartärs in Mitteleuropa. Tübinger Monographien zur Urgeschichte 11: 437- 448 (Festschrift Müller-Beck); Tübingen.

— \& Rosendahl, W. (1997): Datenauszug zu Megaloceros im Jungpleistozän Europas aus der EUQUAMDatenbank der ESF - Stand März 97; Bonn

Kolstrup, E. (1979): Herbs as july temperature indicators for parts of the pleniglacial and late-glacial in the Netherlands. - Geol. Mijnbouw, 58: 377-380; 's Gravenhage.

Liljergen, R. (1975): Subfossila vertebratfynd fran Skane. - University of Lund, Department of Quarternary Geology, Report 8, 187 S.; Lund.

- Eкsтröм, J. (1996): The terrestrial Late Glacial fauna in South Sweden. - In: Larsson, L. (Hrsg.): The earliest settlement of Scandinavia and its relationsship with neighbouring areas. Acta Archaeologica Lundensia 24: 135-139; Lund.
Lorblanchet, M. (1984): Cougnac. - In: L'Art des Cavernes. Atlas des Grottes Ornées paléolithiques Francaise: 483-487; Paris.

— (1997): Höhlenmalerei - Ein Handbuch. - 340 S.; Sigmaringen.

Mangerud, J., Andersen, S. T., Berglund, B. E. \& Donner, J. J. (1974): Quaternary stratigraphy of Norden, a proposal for terminology and classification. Boreas, 3: 109-128; Oslo.

Moore, P. D., WebB, J. A \& Collinson, M. E. (1991): Pollen analysis. - $216 \mathrm{~S}$.; Oxford.

Morel, P. (1993): Un chasse à l'ours brun il y a 12000 ans: nouvelle découverte à la grotte du Bichon (La Chaux-de-Fonds). - Archäologie der Schweiz 16: 111-117; Basel.

RüHBERG, N. (1987): Die Grundmoräne des jüngsten Weichselvorstoßes im Gebiet der DDR. - Zeitschrift für geologische Wissenschaften 15 (6): 759-767; Berlin.

- Schulz, W., Bülow, W. von, Müller, U., KrienKe, H.D., Bremer, F. \& Dann, T. (1995): Mecklenburg-Vorpommern. - In: BeNDA, L. (Hrsg.): Das Quartär Deutschlands, 95-115; Berlin, Stuttgart.

Schlichting, E., Blume, H.-P. \& Stahr, K. (1995): Bodenkundliches Praktikum. - 295 S.; Berlin.

SkogeN, A. (1972): The Hippophaë rhamnoides alluvial forest at Leinöra, Central Norway. A. phytosociological and ecological study. - K. norske Vidensk. selsk. Skr. 4, 115 S.: Oslo.

Street, M. (1996): The Late Glacial faunal assemblage from Endingen, Lkr. Nordvorpommern. - Archäologisches Korrespondenzblatt 26 (1): 33-42; Mainz.

- \& BaAles, M. (1997): Les gropes a Federmesser de l'Allerød en Rhénanie Centrale (Allemagne). Bulletin de la Société Préhistorique Française, 94: 373-386; Paris.

STUART, A. J. (1991): Mamamalian extinctions in the Late Pleistocene of Northern Eurasiia and North America. Biological Review 66: 453-562.

Terberger, T. (1996a): Die „Riesenhirschfundstelle“ von Endingen, Lkr. Nordvorpommern. Spätglaziale Besiedlungsspuren in Nordostdeutschland. - Archäologisches Korrespondenzblatt 26 (1): 13-32; Mainz.

- (1996b): The erly settlement of North-East Germany (Mecklenburg-Vorpommern). - In: LARsson, L. (Hrsg.): The earliest settlement of Scandinavia and its relationsship with neighbouring areas. Acta Archaeologica Lundensia Ser. 8, 24: 111-122; Stockholm.

- (1997): Zur ältesten Besiedlungsgeschichte Mecklenburg-Vorpommerns. - Archäologische Berichte aus Mecklenburg-Vorpommern, 4: 6-21; Waren/ Müritz.

TGL 25232 (1971): Analyse des Geschiebebestandes quartärer Grundmoränen. - 6 Blätter; Berlin.

Tidelski, F. (1960): Pollenanalytische Untersuchungen von voll-, spät- und postglazialen Ablagerungen aus dem Trentmoor und dem Brennacker (Krs. Plön). - Schriften Naturw. Ver. Schleswig-Holstein, 30: 92- 09; Kiel. 
Trautsch, G. (1958): Geologische Verhältnisse des Endinger Bruchs und seiner Randgebiete. - Unveröff. Diplomarbeit, Universität Greifswald, Geol.Paläont. Inst. 55.; Greifswald.

TuCKER, M. E. (1985): Einführung in die Sedimentpetrographie. - 265 S.; Stuttgart.

Usinger, H. (1985): Pollenstratigraphische, vegetationsund klimageschichtliche Gliederung des „BöllingAlleröd Komplexes" in Schleswig-Holstein und ihre Bedeutung für die Spätglazial-Stratigraphie in benachbarten Gebieten. - FLora, 177: 1 - 43; Berlin.

Wohlfarth, B., Skog, G., Possnert, G. \& Holmquist, B. (1998): Pitfalls in the AMS radiocarbon-dating of terrestrial macrofossils. - Journal of Quaternary Science, 13: 137-145, Oxford.

Woldstedt, P. (1955): Norddeutschland und angrenzende Gebiete im Eiszeitalter. - 467 S.; Stuttgart.

Woodman, P.. McCarthy, M. \& Monaghan, N. (1997): The Irish QUaternary fauna project. Quaternary Science Reviews. 16: 129-159; Oxford.

Manuskript eingegangen am 6. Oktober 1998 